\title{
Chemical markers of possible hot spots on Mars
}

\author{
Ah-San Wong and Sushil K. Atreya \\ Department of Atmospheric, Oceanic, and Space Sciences, University of Michigan, Ann Arbor, Michigan, USA
}

Thérèse Encrenaz

Laboratoire d'Etudes Spatiales et d'Instrumentation en Astrophysique (DESPA), Observatoire de Paris, Meudon, France

Received 16 January 2003; accepted 3 March 2003; published 15 April 2003.

[1] Although there is no evidence of active volcanism on Mars today, "localized" outgassing sources, the hot spots, may not be ruled out. If outgassing does occur somewhere on Mars, water, carbon dioxide, sulfur species, methane, and to a lesser extent, halogens would be the likely molecules of outgassing, based on terrestrial analogs. The sulfur species, methane, and halogens have not been detected in the Martian atmosphere, but the observations were averaged over large areas, which could result in substantial dilution in abundances. If the interpretation of certain Mars Global Surveyor images indicating recent ground water seepage and surface runoff [Malin and Edgett, 2000] is correct, it may imply that Mars could still be active internally in some places from time to time, and outgassing of the abovementioned species may occur with or without the water seepage. Moreover, if the tentative detection of formaldehyde $\left(\mathrm{CH}_{2} \mathrm{O}\right)$ in the equatorial region of Mars [Korablev et al., 1993] is confirmed by future observations, it would imply at least local outgassing of methane, whose oxidation results in the formation of $\mathrm{CH}_{2} \mathrm{O}$. Considering the possibility of outgassing from some localized hot spots, we have developed a one-dimensional photochemical model that includes methane $\left(\mathrm{CH}_{4}\right)$, sulfur dioxide $\left(\mathrm{SO}_{2}\right)$, and hydrogen sulfide $\left(\mathrm{H}_{2} \mathrm{~S}\right)$, starting with their current "global average" upper limits of $0.02,0.1$, and $0.1 \mathrm{ppm}$ at the surface, respectively, and then progressively increasing their abundances above possible hot spots. We find that the introduction of methane into the Martian atmosphere results in the formation of mainly formaldehyde, methyl alcohol $\left(\mathrm{CH}_{3} \mathrm{OH}\right)$, and ethane $\left(\mathrm{C}_{2} \mathrm{H}_{6}\right)$, whereas the introduction of the sulfur species produces mainly sulfur monoxide (SO) and sulfuric acid $\left(\mathrm{H}_{2} \mathrm{SO}_{4}\right)$. The effect of outgassed halogens on the Martian atmosphere is found to be negligible. Depending upon the flux of outgassed molecules from possible hot spots, some of these species and the resulting new molecules may be detectable locally, either by remote sensing or in situ measurements. INDEX TERMS: 5405 Planetology: Solid Surface Planets: Atmospheres-composition and chemistry; 6225 Planetology: Solar System Objects: Mars; 0370 Atmospheric Composition and Structure: Volcanic effects (8409); 0317 Atmospheric Composition and Structure: Chemical kinetic and photochemical properties; 5480 Planetology: Solid Surface Planets: Volcanism (8450); KEYWORDS: Mars, photochemistry, hot spot, outgassing, biomarkers, sulfur dioxide

Citation: Wong, A.-S., S. K. Atreya, and T. Encrenaz, Chemical markers of possible hot spots on Mars, J. Geophys. Res., 108(E4), 5026, doi:10.1029/2002JE002003, 2003.

\section{Introduction}

[2] Based on the record of cratering densities in the calderas and along the flanks of volcanoes, it has been suggested that the volcanoes in the Tharsis region of Mars were active in recent geologic time, perhaps within the past few hundred million years. Although there is no evidence of volcanism on Mars today, outgassing from small, localized sources, the hot spots, may not be ruled out. Typically, carbon dioxide, water vapor, sulfur dioxide $\left(\mathrm{SO}_{2}\right)$, hydrogen sulfide $\left(\mathrm{H}_{2} \mathrm{~S}\right)$, methane $\left(\mathrm{CH}_{4}\right)$, and to a lesser extent,

Copyright 2003 by the American Geophysical Union. 0148-0227/03/2002JE002003 halogens are outgassed from terrestrial hot spots [Walker, 1997]. On Mars, sulfur and halogen species have not been detected in the atmosphere, and only a tentative detection of one hydrocarbon, formaldehyde $\left(\mathrm{CH}_{2} \mathrm{O}, \mathrm{H}_{2} \mathrm{CO}\right)$, has been reported in the equatorial region during northern spring [Korablev et al., 1993]. If the $\mathrm{CH}_{2} \mathrm{O}$ detection is correct, its existence could imply a large flux of methane into the Martian atmosphere. Methane could either be abiotic in nature or could be released as a metabolic by-product by any subsurface bacteria that use the readily available Martian $\mathrm{H}_{2}$ or $\mathrm{CO}$ as an energy source. However, methane has not yet been detected on Mars. The nondetection of methane and other possible outgassing species should be viewed with some caution, as the observations of such species 
represent averages over large areas, typically the disk or the hemisphere of Mars so that small localized outgassing sources, especially if transient, could very well go undetected. Furthermore, if the suggestion of recent ground water seepage and surface runoff, particularly at the middle and high latitudes as interpreted from certain Mars Global Surveyor images [Malin and Edgett, 2000], is correct, this too may imply that even now Mars could be active from time to time at least in some places. With the above in mind, and due to considerable interest in the question of extinct or extant life on Mars, we have developed a one-dimensional photochemical model, considering the possibility that methane, sulfur dioxide, hydrogen sulfide, and halogens from any outgassing sources may be introduced into the Martian atmosphere. From this model we predict the abundances of the various new molecules that are expected to be formed in the ensuing atmospheric chemistry, in the hope of guiding future remote sensing and in situ measurements of the chemical markers of possible hot spots on Mars.

[3] The photochemical model for hot spots discussed in this paper assumes the "normal" Martian atmosphere model of Atreya and $G u$ [1994] as a starting point. In general terms, the Atreya and Gu [1994] model is similar to the models of Krasnopolsky [1993] and Nair et al. [1994]. Krasnopolsky [1993] includes $\mathrm{SO}_{2}$ in his model, but that study is preliminary and considers only a very small amount of $\mathrm{SO}_{2}$ released from the airborne dust. In their one-dimensional, globally averaged photochemical model of Mars, Summers et al. [2002] consider several biogenically produced trace species including $\mathrm{CH}_{4}$ and $\mathrm{H}_{2} \mathrm{~S}$. In this model they calculate, for example, a methane abundance that is roughly comparable to the global average upper limit for this species. In the present work, we introduce $\mathrm{SO}_{2}, \mathrm{H}_{2} \mathrm{~S}$, and $\mathrm{CH}_{4}$ into the Martian atmosphere from localized outgassing sources, the hot spots. We begin with their current global average upper limits and then consider progressively larger abundances over the hot spots. We do not consider halogens in our model. The present global upper limit of hydrogen chloride $\left(2 \times 10^{-9}\right)$ [Krasnopolsky et al., 1997], which is presumably the most abundant halogen, is already much smaller than that of other possible outgassing species. In terrestrial outgassing sources, chlorine compounds $(\mathrm{HCl}$, $\mathrm{Cl}_{2}$, etc.) comprise a small fraction of $\mathrm{SO}_{2}$ or the total atmospheric $\mathrm{CH}_{4}$ [Walker, 1997]. In view of the likely negligible amounts of any outgassed halogens and the fact that even their photolysis would be shielded by the copious amounts of $\mathrm{CO}_{2}$ in the Martian atmosphere, the halogens are not expected to play a significant role in the hot spot chemistry of Mars. In the following paragraphs, we describe first the photochemical model and then discuss the results of numerical computations corresponding to the hot spots.

\section{Photochemical Model}

[4] Photochemical models have been used previously to study the distribution of molecules in the atmosphere of Mars [Krasnopolsky, 1993; Atreya and Gu, 1994; Nair et al., 1994]. In this paper we have developed a new onedimensional photochemical model for Mars to investigate the consequence of possible hot spots. The model solves for each chemical species the steady state continuity-diffusion equations using finite-difference techniques. The density of each species as a function of altitude is calculated from the ground up to $220 \mathrm{~km}$. The solar flux, temperature profile, and eddy diffusion coefficients are all global and seasonal averaged values taken from Atreya and Gu [1994]. The dust optical depth is taken to be 0.4 , which is typical for Mars [Krasnopolsky, 1993]. Water content is the global averaged value of $150 \mathrm{ppm}$ or $10 \mathrm{pr}-\mu \mathrm{m}$ at the surface. The pressure at the surface is $6.10 \mathrm{mbar}$, and the atmospheric density is computed assuming hydrostatic equilibrium. Concentration of $\mathrm{CO}_{2}$ at any given altitude is held fixed. Molecular nitrogen is included as a background gas. The lower boundary conditions are mixing ratios of $2.7 \times 10^{-2}$ for $\mathrm{N}_{2}, 1.50 \times 10^{-4}$ for $\mathrm{H}_{2} \mathrm{O}$, and fixed concentration of $\mathrm{CO}_{2}$ based on surface pressure and temperature. Upper boundary conditions are $\mathrm{H}$ and $\mathrm{H}_{2}$ effusion velocities of $3.1 \times 10^{3}$ and $3.4 \times 10^{1} \mathrm{~cm} \mathrm{~s}^{-1}$, respectively, and escape flux of atomic oxygen of $1.2 \times 10^{8} \mathrm{~cm}^{-2} \mathrm{~s}^{-1}$ [Nair et al., 1994] to balance the escape rate of hydrogen. Fluxes of all other species are zero at both boundaries. Condensation of $\mathrm{H}_{2} \mathrm{O}$ and $\mathrm{H}_{2} \mathrm{O}_{2}$ are considered; however, in the present model $\mathrm{H}_{2} \mathrm{O}_{2}$ is always found to be subsaturated. The basic photochemical reactions for the normal, i.e., non-hot spot, atmosphere are listed in Table 1 .

[5] New species and reactions added for calculation of hot spot chemistry are described in the next three sections. Since the photolysis of methane is spectrally and spatially removed from the photolysis of $\mathrm{SO}_{2}$ and $\mathrm{H}_{2} \mathrm{~S}$, we find that there is little significant interaction between the sulfur species and the hydrocarbon species. This is further amplified by the fact the $\mathrm{S}$ abundance is extremely low at all altitudes to permit any appreciable product density upon reaction with the hydrocarbons, even if the (unmeasured) rate of $\mathrm{S}+\mathrm{CH}_{3}$ reaction were fast (other sulfur reactions were found to be even less important). In view of the above, we consider the hydrocarbon and the sulfur chemical schemes separately.

\section{Sulfur Chemistry}

[6] Farquhar et al. [2000] and Wänke and Dreibus [1994] have speculated on the presence of $\mathrm{SO}_{2}$ and $\mathrm{H}_{2} \mathrm{~S}$ in the atmosphere of Mars, generally in the past. In the current work, we investigate in detail the photochemistry of sulfur species in Martian atmosphere. The main reactions of sulfur chemistry and their rate coefficients are listed in Table 2. The principal reaction pathways are illustrated in Figure 1 and will be discussed later in this section.

[7] The upper limits of $\mathrm{SO}_{2}$ and $\mathrm{H}_{2} \mathrm{~S}$ are $0.1 \mathrm{ppm}$ each for globally averaged observations [Maguire, 1977]. In the model we first study the photochemistry of each of these two gases as independent outgassing species. We start with $\mathrm{SO}_{2}$ as the only outgassed species, with $0.1 \mathrm{ppm}$ at the surface. In subsequent model runs we increase its concentration gradually until it reaches $10 \mathrm{ppm}$ above the hot spot. We do the same with $\mathrm{H}_{2} \mathrm{~S}$ outgassing by itself. Then we assume both gases outgassing from the surface together and increase the concentration of each from $0.1 \mathrm{ppm}$ to $100 \mathrm{ppm}$. Since the existence and nature of hot spots are speculative, we consider enhancement by up to a factor of 1000 over outgassing sources relative to the global upper limits of $\mathrm{H}_{2} \mathrm{~S}$ and $\mathrm{SO}_{2}$. However, enhancement factors are necessarily arbitrary. They could be lower or even greater, depending 
Table 1. Basic Set of Photochemical Reactions Along With Their Rate Coefficients and References Used in the Model

\begin{tabular}{|c|c|c|c|}
\hline & Reaction & Rate Coefficient $\mathrm{t}^{\mathrm{a}}$ & Reference \\
\hline (R1) & $\mathrm{CO}_{2}+\mathrm{h} \nu \rightarrow \mathrm{CO}+\mathrm{O} \rightarrow \mathrm{CO}+\mathrm{O}\left({ }^{1} \mathrm{D}\right)$ & $\begin{aligned} J\left(\mathrm{CO}_{2}\right)= & 1.4 \times 10^{-12} \\
& 7.9 \times 10^{-7}\end{aligned}$ & $\begin{array}{l}\text { Shemansky [1972], Lewis and } \\
\text { Carver [1983], Atreya } \\
\text { and } G u[1994]\end{array}$ \\
\hline (R2) & $\mathrm{O}_{2}+\mathrm{h} \nu \rightarrow 2 \mathrm{O} \rightarrow \mathrm{O}\left({ }^{1} \mathrm{D}\right)+\mathrm{O}$ & $\begin{aligned} & J\left(\mathrm{O}_{2}\right)= 8.9 \times 10^{-11} \\
& 9.0 \times 10^{-7}\end{aligned}$ & Watanabe et al. [1953] \\
\hline (R3) & $\mathrm{O}_{3}+\mathrm{h} \nu \rightarrow \mathrm{O}_{2}+\mathrm{O}\left({ }^{1} \mathrm{D}\right) \rightarrow \mathrm{O}_{2}+\mathrm{O}$ & $\begin{aligned} J\left(\mathrm{O}_{3}\right)= & 6.0 \times 10^{-5} \\
1.7 & \times 10^{-3}\end{aligned}$ & $\begin{array}{l}\text { World Meteorological } \\
\text { Organization (WMO) [1985] }\end{array}$ \\
\hline (R4) & $\mathrm{H}_{2} \mathrm{O}+\mathrm{h} \nu \rightarrow \mathrm{OH}+\mathrm{H}$ & $\begin{aligned} J\left(\mathrm{H}_{2} \mathrm{O}\right)= & 2.0 \times 10^{-10} \\
& 1.6 \times 10^{-6}\end{aligned}$ & Thompson et al. [1963] \\
\hline (R5) & $\mathrm{HO}_{2}+\mathrm{h} \nu \rightarrow \mathrm{OH}+\mathrm{O}$ & $\begin{aligned} J\left(\mathrm{HO}_{2}\right)= & 3.6 \times 10^{-6} \\
& 1.9 \times 10^{-4}\end{aligned}$ & Okabe [1978] \\
\hline (R6) & $\mathrm{H}_{2} \mathrm{O}_{2}+\mathrm{h} \nu \rightarrow 2 \mathrm{OH}$ & $\begin{aligned} J\left(\mathrm{H}_{2} \mathrm{O}_{2}\right)= & 3.3 \times 10^{-6} \\
& 2.3 \times 10^{-5}\end{aligned}$ & Okabe [1978] \\
\hline (R7) & $\mathrm{O}+\mathrm{O}+\mathrm{M} \rightarrow \mathrm{O}_{2}+\mathrm{M}$ & $5.21 \times 10^{-35} \mathrm{e}^{900 / \mathrm{T}}$ & Tsang and Hampson [1986] \\
\hline (R8) & $\mathrm{O}\left({ }^{1} \mathrm{D}\right)+\mathrm{CO}_{2} \rightarrow \mathrm{O}+\mathrm{CO}_{2}$ & $7.41 \times 10^{-11} \mathrm{e}^{120 / \mathrm{T}}$ & DeMore et al. [1997] \\
\hline (R9) & $\mathrm{O}_{2}+\mathrm{O}+\mathrm{M} \rightarrow \mathrm{O}_{3}+\mathrm{M}$ & $2.95 \times 10^{-28} \mathrm{~T}^{-2.3}$ & DeMore et al. [1997] for $\mathrm{N}_{2}$ \\
\hline (R10) & $\mathrm{O}_{3}+\mathrm{O} \rightarrow 2 \mathrm{O}_{2}$ & $8.2 \times 10^{-12} \mathrm{e}^{-2060 / \mathrm{T}}$ & Atkinson et al. [1997] \\
\hline (R11) & $\mathrm{H}+\mathrm{O}_{2}+\mathrm{M} \rightarrow \mathrm{HO}_{2}+\mathrm{M}$ & $5.16 \times 10^{-28} \mathrm{~T}^{-1.6}$ & DeMore et al. [1992] \\
\hline (R12) & $\mathrm{H}+\mathrm{O}_{3} \rightarrow \mathrm{OH}+\mathrm{O}_{2}$ & $1.4 \times 10^{-10} \mathrm{e}^{-470 / \mathrm{T}}$ & DeMore et al. [1992] \\
\hline (R13) & $\mathrm{H}+\mathrm{H}+\mathrm{M} \rightarrow \mathrm{H}_{2}+\mathrm{M}$ & $2.7 \times 10^{-31} \mathrm{~T}^{-0.6}$ & Baulch et al. [1992] \\
\hline (R14) & $\mathrm{H}_{2}+\mathrm{O}\left({ }^{1} \mathrm{D}\right) \rightarrow \mathrm{OH}+\mathrm{H}$ & $1.0 \times 10^{-10}$ & Gericke and Comes [1981] \\
\hline (R15) & $\mathrm{H}_{2} \mathrm{O}+\mathrm{O}\left({ }^{1} \mathrm{D}\right) \rightarrow 2 \mathrm{OH}$ & $2.2 \times 10^{-10}$ & DeMore et al. [1992] \\
\hline (R16) & $\mathrm{OH}+\mathrm{CO} \rightarrow \mathrm{CO}_{2}+\mathrm{H}$ & $1.99 \times 10^{-17} \mathrm{~T}^{1.35} \mathrm{e}^{365 / \mathrm{T}}$ & Larson et al. [1988] \\
\hline (R17) & $\mathrm{OH}+\mathrm{O} \rightarrow \mathrm{O}_{2}+\mathrm{H}$ & $2.2 \times 10^{-11} \mathrm{e}^{120 / \mathrm{T}}$ & DeMore et al. [1992] \\
\hline (R18) & $\mathrm{OH}+\mathrm{O}_{3} \rightarrow \mathrm{O}_{2}+\mathrm{HO}_{2}$ & $1.6 \times 10^{-12} \mathrm{e}^{-940 / \mathrm{T}}$ & DeMore et al. [1997] \\
\hline (R19) & $\mathrm{HO}_{2}+\mathrm{O} \rightarrow \mathrm{OH}+\mathrm{O}_{2}$ & $2.9 \times 10^{-11} \mathrm{e}^{200 / \mathrm{T}}$ & Atkinson et al. [1989] \\
\hline (R20) & $\mathrm{HO}_{2}+\mathrm{O}_{3} \rightarrow 2 \mathrm{O}_{2}+\mathrm{OH}$ & $1.1 \times 10^{-14} \mathrm{e}^{-500 / \mathrm{T}}$ & DeMore et al. [1997] \\
\hline (R21) & $\mathrm{HO}_{2}+\mathrm{OH} \rightarrow \mathrm{O}_{2}+\mathrm{H}_{2} \mathrm{O}$ & $4.8 \times 10^{-11} \mathrm{e}^{250 / \mathrm{T}}$ & DeMore et al. [1997] \\
\hline \multirow[t]{3}{*}{ (R22) } & $\mathrm{HO}_{2}+\mathrm{H} \rightarrow 2 \mathrm{OH}$ & $7.2 \times 10^{-11}$ & Atkinson et al. [1989] \\
\hline & $\rightarrow \mathrm{H}_{2}+\mathrm{O}_{2}$ & $5.6 \times 10^{-12}$ & Atkinson et al. [1989] \\
\hline & $\rightarrow \mathrm{H}_{2} \mathrm{O}+\mathrm{O}$ & $2.4 \times 10^{-12}$ & Atkinson et al. [1989] \\
\hline (R23) & $\mathrm{HO}_{2}+\mathrm{HO}_{2} \rightarrow \mathrm{H}_{2} \mathrm{O}_{2}+\mathrm{O}_{2}$ & $1.5 \times 10^{-12} \mathrm{e}^{-12 / \mathrm{T}}$ & Christensen et al. [2002] \\
\hline (R24) & $\mathrm{H}_{2} \mathrm{O}_{2}+\mathrm{OH} \rightarrow \mathrm{H}_{2} \mathrm{O}+\mathrm{HO}_{2}$ & $2.91 \times 10^{-12} \mathrm{e}^{-161 / \mathrm{T}}$ & DeMore et al. [1992] \\
\hline (R25) & $\mathrm{N}_{2}+\mathrm{O}\left({ }^{1} D\right) \rightarrow \mathrm{N}_{2}+\mathrm{O}$ & $1.8 \times 10^{-11} \mathrm{e}^{110 / \mathrm{T}}$ & DeMore et al. [1997] \\
\hline
\end{tabular}

Photolysis rate coefficients are given at $10 \mathrm{~km}$ and $200 \mathrm{~km}$.

upon the size, strength, and the duration of the source. For example, gases released from a $1 \mathrm{~km}$ diameter source may be diluted by a factor of as much as $10^{8}$ when spread uniformly over the Martian surface. The results of a few investigative runs are summarized in Table 3.

[8] Models $\mathrm{A}$ and $\mathrm{B}$ are cases with only $\mathrm{SO}_{2}$ outgassing, with $0.1 \mathrm{ppm}$ and $10 \mathrm{ppm}$ mixing ratio at the surface, respectively. Models $\mathrm{C}$ and $\mathrm{D}$ are cases with only $\mathrm{H}_{2} \mathrm{~S}$ outgassing, $0.1 \mathrm{ppm}$ and $10 \mathrm{ppm}$ surface mixing ratio, respectively. Models E, F, and G correspond to the cases where both gases are outgassed, with $0.1 \mathrm{ppm}, 10 \mathrm{ppm}$, and $100 \mathrm{ppm}$ surface mixing ratio of each species, respectively. Only for the purpose of illustration, the result of a "nominal" case, Model G, is summarized here. The resulting mixing ratios of $\mathrm{SO}_{2}, \mathrm{H}_{2} \mathrm{~S}$, and $\mathrm{SO}$ at $10 \mathrm{~km}$ are $1.7 \times 10^{-4}$, $1.8 \times 10^{-5}$, and $1.7 \times 10^{-7}$, respectively (see Table 3 ). These are the most abundant species and possibly detectable. The number densities and mixing ratios of important sulfur species are plotted in Figure 2.

[9] Once in the atmosphere, sulfur dioxide is photodissociated to produce sulfur monoxide (SO). Sulfur monoxide can be photodissociated to form sulfur atoms. The reaction of $\mathrm{SO}$ with $\mathrm{O}_{2}$ or $\mathrm{OH}$ recycles sulfur atoms back to $\mathrm{SO}_{2}$ (R33, R35), while the reaction with ozone $\left(\mathrm{O}_{3}\right)$ forms sulfur trioxide $\left(\mathrm{SO}_{3}, \mathrm{R} 34\right)$. Sulfur trioxide quickly combines with water vapor to form sulfuric acid $\left(\mathrm{H}_{2} \mathrm{SO}_{4}, \mathrm{R} 39\right)$.
The saturation vapor pressure measurements of $\mathrm{H}_{2} \mathrm{SO}_{4}$ [Stull, 1947] can be fitted by the following expression: $\log _{10} \mathrm{P}\left(\mathrm{H}_{2} \mathrm{SO}_{4}\right)=-3954.90 / \mathrm{T}+9.4570$, for temperature range approximately between $420 \mathrm{~K}$ and $580 \mathrm{~K}$, where $\mathrm{P}\left(\mathrm{H}_{2} \mathrm{SO}_{4}\right)$ is the saturation vapor pressure of $\mathrm{H}_{2} \mathrm{SO}_{4}$ in $\mathrm{mmHg}$. Extrapolating this expression to the lower temperatures of the Martian atmosphere, it is found that the $\mathrm{H}_{2} \mathrm{SO}_{4}$ produced from photochemistry would condense in the lower atmosphere. For example, $\mathrm{P}\left(\mathrm{H}_{2} \mathrm{SO}_{4}\right)=4.8 \times 10^{-11} \mathrm{mmHg}$ at $200 \mathrm{~K}$, an extremely low value. Without condensation, $\mathrm{H}_{2} \mathrm{SO}_{4}$ mixing ratio reaches a value of approximately $40 \mathrm{ppm}$ in Model $\mathrm{G}$ where $\mathrm{SO}_{2}$ and $\mathrm{H}_{2} \mathrm{~S}$ are each 100 ppm above the hot spot. Taking condensation into account, the resulting column abundance of $\mathrm{H}_{2} \mathrm{SO}_{4}$ above $10 \mathrm{~km}$ is $2.1 \times 10^{12} \mathrm{~cm}^{-2}$, or approximately $0.2 \mathrm{ppb}$, too low to be plotted in Figure 2. However, the actual $\mathrm{H}_{2} \mathrm{SO}_{4}$ vapormixing ratio would depend on a number of factors, including the abundance of water, concentration of sulfuric acid, degree of supersaturation, and the range of validity of the saturation vapor pressure relationship.

[10] We have found that the outgassing of $\mathrm{H}_{2} \mathrm{~S}$ alone gives similar composition as the outgassing of $\mathrm{SO}_{2}$ (see Table 3). This is due to the fact that the outgassed $\mathrm{H}_{2} \mathrm{~S}$ is rapidly converted to $\mathrm{SO}_{2}$ (see Figure 1). After $\mathrm{H}_{2} \mathrm{~S}$ is released into the atmosphere, it undergoes photodissociation to form mercapto radical HS (R28). The photodissociation 
Table 2. Photochemical Reactions of Sulfur Species Along With Their Rate Coefficients and References Studied in the Model

\begin{tabular}{|c|c|c|c|}
\hline & Reaction & Rate Coefficient $^{\mathrm{a}}$ & Reference \\
\hline (R26) & $\mathrm{SO}+\mathrm{h} \nu \rightarrow \mathrm{S}+\mathrm{O}$ & $\begin{aligned} J(\mathrm{SO})= & 7.4 \times 10^{-9} \\
& 8.6 \times 10^{-5}\end{aligned}$ & Phillips [1981] \\
\hline (R27) & $\mathrm{SO}_{2}+\mathrm{h} \nu \rightarrow \mathrm{SO}+\mathrm{O}$ & $\begin{aligned} J\left(\mathrm{SO}_{2}\right)= & 6.1 \times 10^{-18} \\
& 4.5 \times 10^{-5}\end{aligned}$ & Okabe [1971], Welge [1974] \\
\hline (R28) & $\begin{aligned} \mathrm{SO}_{3}+\mathrm{h} v & \rightarrow \mathrm{SO}_{2}+\mathrm{O} \\
& \rightarrow \mathrm{SO}_{2}+\mathrm{O}\left({ }^{1} \mathrm{D}\right)\end{aligned}$ & $\begin{aligned} J\left(\mathrm{SO}_{3}\right)= & 1.2 \times 10^{-7} \\
& 2.1 \times 10^{-4}\end{aligned}$ & Okabe [1978] \\
\hline (R29) & $\mathrm{H}_{2} \mathrm{~S}+\mathrm{h} \nu \rightarrow \mathrm{HS}+\mathrm{H}$ & $\begin{aligned} J\left(\mathrm{H}_{2} \mathrm{~S}\right)= & 1.7 \times 10^{-6} \\
& 7.2 \times 10^{-5}\end{aligned}$ & Okabe [1978] \\
\hline (R30) & $\mathrm{S}+\mathrm{O}_{2} \rightarrow \mathrm{SO}+\mathrm{O}$ & $2.3 \times 10^{-12}$ & DeMore et al. [1997] \\
\hline (R31) & $\mathrm{S}+\mathrm{O}_{3} \rightarrow \mathrm{SO}+\mathrm{O}_{2}$ & $1.2 \times 10^{-11}$ & DeMore et al. [1997] \\
\hline (R32) & $\mathrm{SO}+\mathrm{O}+M \rightarrow \mathrm{SO}_{2}+M$ & $5.10 \times 10^{-31}$ & Singleton and Cvetanovic $[1988]$ \\
\hline (R33) & $\mathrm{SO}+\mathrm{O}_{2} \rightarrow \mathrm{SO}_{2}+\mathrm{O}$ & $2.6 \times 10^{-13}$ & DeMore et al. [1997] \\
\hline (R34) & $\mathrm{SO}+\mathrm{O}_{3} \rightarrow \mathrm{SO}_{3}+\mathrm{O}_{2}$ & $3.6 \times 10^{-12}$ & DeMore et al. [1997] \\
\hline (R35) & $\mathrm{SO}+\mathrm{OH} \rightarrow \mathrm{SO}_{2}+\mathrm{H}$ & $8.59 \times 10^{-11}$ & DeMore et al. [1997] \\
\hline (R36) & $\mathrm{SO}+\mathrm{SO} \rightarrow \mathrm{SO}_{2}+\mathrm{S}$ & $5.8 \times 10^{-12} \mathrm{e}^{-1760 / \mathrm{T}}$ & Yung and DeMore [1999] \\
\hline (R37) & $\mathrm{SO}_{2}+\mathrm{O}+M \rightarrow \mathrm{SO}_{3}+M$ & $1.61 \times 10^{-42} \mathrm{~T}^{3.6}$ & DeMore et al. [1997] \\
\hline (R38) & $\mathrm{SO}_{2}+\mathrm{OH}+M \rightarrow \mathrm{HSO}_{3}+M$ & $4.39 \times 10^{-23} \mathrm{~T}^{-3.3}$ & DeMore et al. [1997] \\
\hline (R39) & $\mathrm{SO}_{3}+\mathrm{H}_{2} \mathrm{O} \rightarrow \mathrm{H}_{2} \mathrm{SO}_{4}$ & $1.20 \times 10^{-15}$ & Reiner and Arnold [1994] \\
\hline (R40) & $\mathrm{SO}_{3}+\mathrm{SO} \rightarrow 2 \mathrm{SO}_{2}$ & $1.99 \times 10^{-15}$ & Chung et al. [1975] \\
\hline (R41) & $\mathrm{HSO}_{3}+\mathrm{O}_{2} \rightarrow \mathrm{SO}_{3}+\mathrm{HO}_{2}$ & $1.3 \times 10^{-12} \mathrm{e}^{-330 / \mathrm{T}}$ & DeMore et al. [1997] \\
\hline (R42) & $\mathrm{HS}+\mathrm{O} \rightarrow \mathrm{SO}+\mathrm{H}$ & $1.60 \times 10^{-10}$ & DeMore et al. [1997] \\
\hline (R43) & $\mathrm{HS}+\mathrm{O}_{3} \rightarrow \mathrm{HSO}+\mathrm{O}_{2}$ & $9.00 \times 10^{-12} \mathrm{e}^{-280 / \mathrm{T}}$ & DeMore et al. [1997] \\
\hline (R44) & $\mathrm{HS}+\mathrm{H} \rightarrow \mathrm{S}+\mathrm{H}_{2}$ & $2.16 \times 10^{-11}$ & Nicholas et al. [1979] \\
\hline (R45) & $\mathrm{HS}+\mathrm{HO}_{2} \rightarrow \mathrm{HSO}+\mathrm{OH}$ & $1.0 \times 10^{-11}$ & Stachnik and Molina [1987] \\
\hline (R46) & $\mathrm{HS}+\mathrm{HS} \rightarrow \mathrm{H}_{2} \mathrm{~S}+\mathrm{S}$ & $4.00 \times 10^{-11}$ & Stachnik and Molina [1987] \\
\hline (R47) & $\mathrm{H}_{2} \mathrm{~S}+\mathrm{O} \rightarrow \mathrm{HS}+\mathrm{OH}$ & $9.21 \times 10^{-12}$ & DeMore et al. [1997] \\
\hline (R48) & $\mathrm{H}_{2} \mathrm{~S}+\mathrm{H} \rightarrow \mathrm{HS}+\mathrm{H}_{2}$ & $1.96 \times 10^{-17} \mathrm{~T}^{2.10} \mathrm{e}^{-352 / \mathrm{T}}$ & Yoshimura et al. [1992] \\
\hline (R49) & $\mathrm{H}_{2} \mathrm{~S}+\mathrm{OH} \rightarrow \mathrm{HS}+\mathrm{H}_{2} \mathrm{O}$ & $6.00 \times 10^{-12} \mathrm{e}^{-75 / \mathrm{T}}$ & DeMore et al. [1997] \\
\hline (R50) & $\mathrm{HSO}+\mathrm{O}_{2} \rightarrow \mathrm{SO}_{2}+\mathrm{OH}$ & $1.7 \times 10^{-15}$ & Bulatov et al. [1986] \\
\hline (R51) & $\mathrm{HSO}+\mathrm{O}_{3} \rightarrow \mathrm{HS}+2 \mathrm{O}_{2}$ & $2.54 \times 10^{-13} \mathrm{e}^{-384 / \mathrm{T}}$ & Wang and Howard [1990] \\
\hline
\end{tabular}

${ }^{\mathrm{a}}$ Units are $\mathrm{s}^{-1}$ for photolysis reactions, $\mathrm{cm}^{3} \mathrm{~s}^{-1}$ for two-body reactions, and $\mathrm{cm}^{6} \mathrm{~s}^{-1}$ for three-body reactions. Photolysis rate coefficients are given at $10 \mathrm{~km}$ and $200 \mathrm{~km}$.

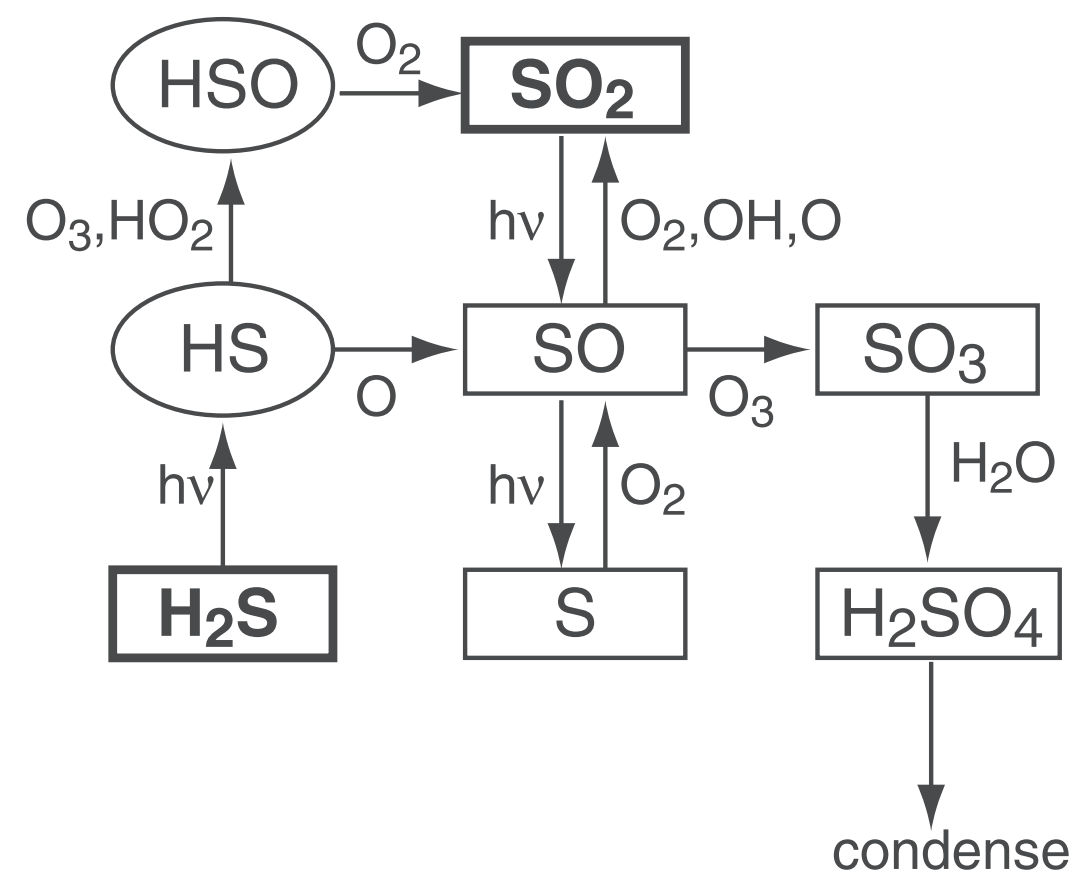

Figure 1. Schematic diagram illustrating the important reaction pathways of sulfur species in the Martian atmosphere. Stable species are in rectangles, radicals are in ovals, and outgassing species are in bold. 
Table 3. Model Results for Sulfur Chemistry in the Martian Atmosphere ${ }^{\mathrm{a}}$

\begin{tabular}{|c|c|c|c|c|c|c|c|c|}
\hline \multirow[b]{3}{*}{ Model } & \multirow{2}{*}{\multicolumn{2}{|c|}{$\begin{array}{c}\text { Outgassing Species } \\
\text { Surface Mixing } \\
\end{array}$}} & \multicolumn{6}{|c|}{ Major Photochemical Products } \\
\hline & & & \multicolumn{2}{|c|}{$\mathrm{SO}_{2}$} & \multicolumn{2}{|c|}{$\mathrm{H}_{2} \mathrm{~S}$} & \multicolumn{2}{|c|}{$\mathrm{SO}$} \\
\hline & $\mathrm{SO}_{2}$ & $\mathrm{H}_{2} \mathrm{~S}$ & Mixing & Column & Mixing & Column & Mixing & Column \\
\hline A & $10^{-7}$ & 0 & $7.4 \times 10^{-8}$ & $5.4 \times 10^{15}$ & 0 & 0 & $7.1 \times 10^{-10}$ & $9.6 \times 10^{14}$ \\
\hline $\mathrm{B}$ & $10^{-5}$ & 0 & $9.1 \times 10^{-6}$ & $7.2 \times 10^{17}$ & 0 & 0 & $1.7 \times 10^{-10}$ & $2.4 \times 10^{16}$ \\
\hline $\mathrm{C}$ & 0 & $10^{-7}$ & $6.0 \times 10^{-8}$ & $5.4 \times 10^{15}$ & $2.1 \times 10^{-8}$ & $7.4 \times 10^{14}$ & $6.7 \times 10^{-10}$ & $1.1 \times 10^{15}$ \\
\hline $\mathrm{D}$ & 0 & $10^{-5}$ & $6.7 \times 10^{-6}$ & $7.7 \times 10^{17}$ & $2.9 \times 10^{-6}$ & $1.3 \times 10^{17}$ & $9.1 \times 10^{-9}$ & $3.1 \times 10^{16}$ \\
\hline $\mathrm{E}$ & $10^{-7}$ & $10^{-7}$ & $1.4 \times 10^{-7}$ & $1.1 \times 10^{16}$ & $2.3 \times 10^{-8}$ & $7.9 \times 10^{14}$ & $1.3 \times 10^{-9}$ & $2.0 \times 10^{15}$ \\
\hline $\mathrm{F}$ & $10^{-5}$ & $10^{-5}$ & $1.7 \times 10^{-5}$ & $1.7 \times 10^{18}$ & $3.0 \times 10^{-6}$ & $1.3 \times 10^{17}$ & $9.1 \times 10^{-9}$ & $4.1 \times 10^{16}$ \\
\hline $\mathrm{G}$ & $10^{-4}$ & $10^{-4}$ & $1.7 \times 10^{-4}$ & $1.8 \times 10^{19}$ & $1.8 \times 10^{-5}$ & $7.4 \times 10^{17}$ & $1.7 \times 10^{-7}$ & $7.8 \times 10^{16}$ \\
\hline \multicolumn{3}{|c|}{ Photochemical lifetime } & \multicolumn{2}{|c|}{$1.4 \times 10^{7} \mathrm{~s}(180$ days $)$} & \multicolumn{2}{|c|}{$7.9 \times 10^{5} \mathrm{~s}(9$ days $)$} & \multicolumn{2}{|c|}{$1.7 \times 10^{4} \mathrm{~s}(4.6$ hours $)$} \\
\hline
\end{tabular}

a'Mixing ratios (labeled "mixing") and the column abundances (in $\mathrm{cm}^{-2}$, labeled "column") are compared at $10 \mathrm{~km}$ in this listing. Lifetime of a species indicates the time to destroy all of that species in the atmosphere photochemically.
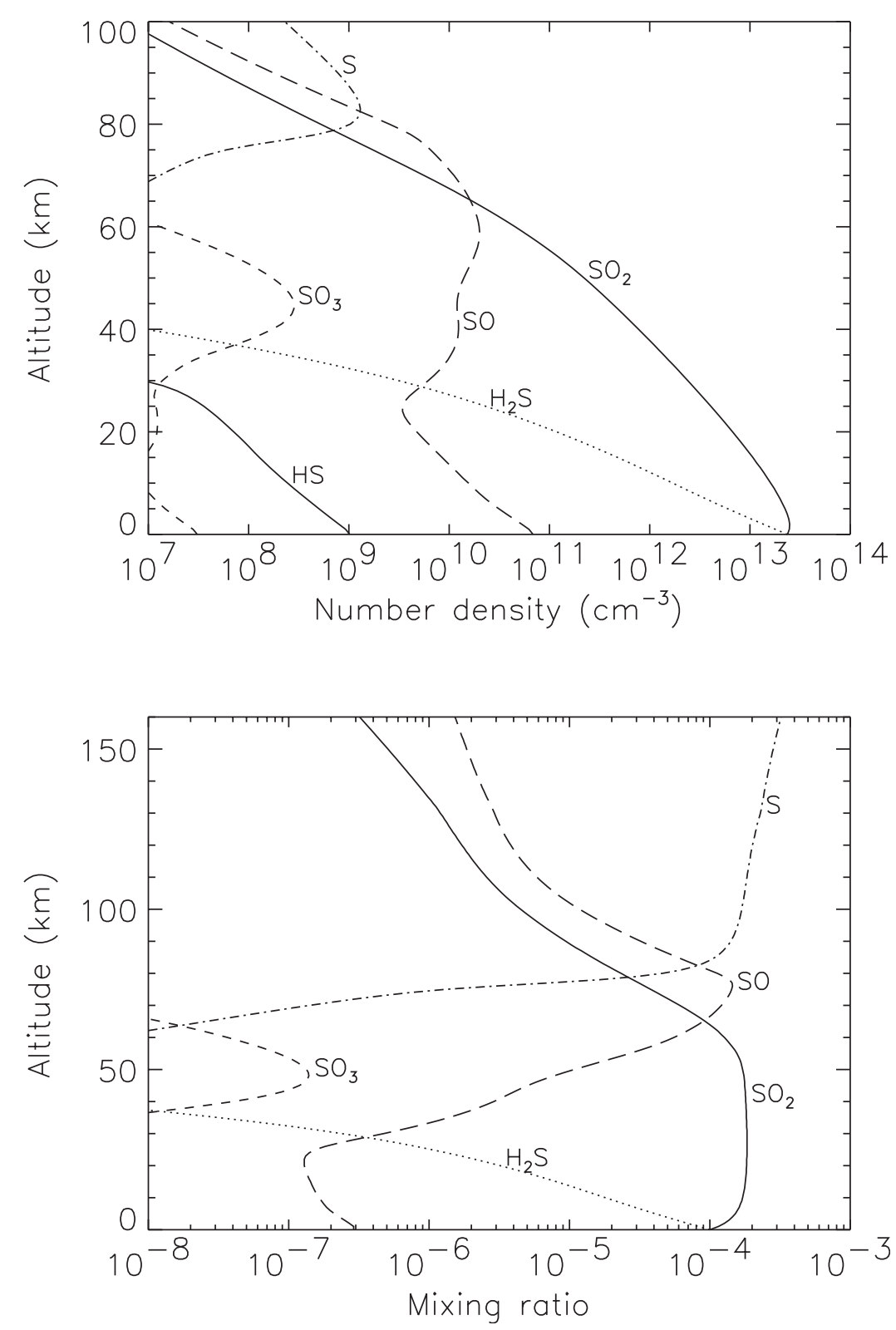

Figure 2. Number densities and mixing ratios versus altitude for important species calculated with $100 \mathrm{ppm}$ each of $\mathrm{H}_{2} \mathrm{~S}$ and $\mathrm{SO}_{2}$ at the surface of Mars (Model G). 
Table 4. Photochemical Reactions of Hydrocarbon Species Along With Their Rate Coefficients and References Studied in the Model

\begin{tabular}{|c|c|c|c|}
\hline & Reaction & Rate Coefficient ${ }^{\mathrm{a}}$ & Reference \\
\hline (R52) & $\begin{aligned} \mathrm{CH}_{4}+\mathrm{h} v & \rightarrow \mathrm{CH}_{3}+\mathrm{H} \\
& \rightarrow{ }^{3} \mathrm{CH}_{2}+2 \mathrm{H} \\
& \rightarrow{ }^{1} \mathrm{CH}_{2}+\mathrm{H}_{2} \\
& \rightarrow{ }^{1} \mathrm{CH}_{2}+2 \mathrm{H} \\
& \rightarrow \mathrm{CH}+\mathrm{H}+\mathrm{H}_{2}\end{aligned}$ & $J\left(\mathrm{CH}_{4}\right)=0,1.7 \times 10^{-6}$ & $\begin{array}{l}\text { Gladstone et al. }[1996], \\
\text { Mordaunt et al. [1993], } \\
\text { Heck et al. }[1996]\end{array}$ \\
\hline (R53) & $\mathrm{HCO}+\mathrm{h} v \rightarrow \mathrm{CO}+\mathrm{H}$ & $\begin{aligned} J(\mathrm{HCO})= & 6.9 \times 10^{-10}, \\
& 6.0 \times 10^{-8}\end{aligned}$ & $\begin{array}{l}\text { König et al. [1984], } \\
\text { Hochanadel et al. [1980], } \\
\text { Okabe [1978] }\end{array}$ \\
\hline (R54) & $\begin{aligned} \mathrm{CH}_{2} \mathrm{O}+\mathrm{h} \nu & \rightarrow \mathrm{HCO}+\mathrm{H} \\
& \rightarrow \mathrm{CO}+\mathrm{H}_{2} \\
& \rightarrow \mathrm{CO}+2 \mathrm{H}\end{aligned}$ & $\begin{aligned} J\left(\mathrm{CH}_{2} \mathrm{O}\right)= & 2.5 \times 10^{-5} \\
& 4.6 \times 10^{-5}\end{aligned}$ & $\begin{array}{l}\text { DeMore et al. }[1992,1990,1987,1985], \\
\text { Suto et al. }[1986], \\
\text { Okabe }[1978], \\
\text { Glicker and Stief }[1971], \\
\text { Mentall et al. }[1971], \\
\text { Gentieu and Mentall }[1970]\end{array}$ \\
\hline (R55) & $\begin{aligned} \mathrm{CH}_{3} \mathrm{OH}+\mathrm{h} v & \rightarrow \mathrm{CH}_{3}+\mathrm{OH} \\
& \rightarrow \mathrm{CH}_{2} \mathrm{O}+\mathrm{H}_{2} \\
& \rightarrow \mathrm{CH}_{3} \mathrm{O}+\mathrm{H}\end{aligned}$ & $\begin{aligned} J\left(\mathrm{CH}_{3} \mathrm{OH}\right)= & 4.7 \times 10^{-8} \\
& 1.8 \times 10^{-6}\end{aligned}$ & $\begin{array}{l}\text { Satyapal and Bersohn [1989], } \\
\text { Nee et al. [1985], Person and } \\
\text { Nicole [1971], } \\
\text { Hagége et al. }[1968]\end{array}$ \\
\hline (R56) & $\begin{aligned} \mathrm{C}_{2} \mathrm{H}_{4}+\mathrm{h} v & \rightarrow \mathrm{C}_{2} \mathrm{H}_{2}+\mathrm{H}_{2} \\
& \rightarrow \mathrm{C}_{2} \mathrm{H}_{2}+2 \mathrm{H}\end{aligned}$ & $\begin{aligned} J\left(\mathrm{C}_{2} \mathrm{H}_{4}\right)= & 3.3 \times 10^{-9} \\
& 6.9 \times 10^{-6}\end{aligned}$ & $\begin{array}{l}\text { Gladstone et al. [1996], } \\
\text { Balko et al. [1992] }\end{array}$ \\
\hline (R57) & $\begin{aligned} \mathrm{C}_{2} \mathrm{H}_{6}+\mathrm{h} & \\
& \rightarrow \mathrm{C}_{2} \mathrm{H}_{2}+2 \mathrm{H}_{2} \\
& \rightarrow \mathrm{C}_{2} \mathrm{H}_{4}+\mathrm{H}_{2} \\
& \rightarrow \mathrm{C}_{2} \mathrm{H}_{4}+2 \mathrm{H} \\
& \rightarrow \mathrm{CH}_{4}+{ }^{1} \mathrm{CH}_{2} \\
& \rightarrow \mathrm{CH}_{3}+\mathrm{CH}_{3}\end{aligned}$ & $J\left(\mathrm{C}_{2} \mathrm{H}_{6}\right)=0,1.4 \times 10^{-6}$ & Gladstone et al. [1996] \\
\hline (R58) & $\mathrm{H}+\mathrm{CO}+\mathrm{M} \rightarrow \mathrm{HCO}+\mathrm{M}$ & $5.30 \times 10^{-34} \mathrm{e}^{-370 / \mathrm{T}}$ & Baulch et al. [1994] \\
\hline (R59) & $\begin{aligned} \mathrm{CH}+\mathrm{O}_{2} & \rightarrow \mathrm{CO}+\mathrm{OH} \\
& \rightarrow \mathrm{HCO}+\mathrm{O}\end{aligned}$ & $\begin{array}{l}2.75 \times 10^{-11} \\
2.75 \times 10^{-11}\end{array}$ & $\begin{array}{l}\text { Baulch et al. }[1992] \\
\text { Baulch et al. }[1992]\end{array}$ \\
\hline (R60) & $\mathrm{CH}+\mathrm{H}_{2} \rightarrow \mathrm{CH}_{3}$ & $8.55 \times 10^{-11} \mathrm{~T}^{0.15}$ & Fulle and Hippler [1997] \\
\hline (R61) & $\mathrm{CH}+\mathrm{H}_{2} \mathrm{O} \rightarrow \mathrm{CH}_{2} \mathrm{OH}$ & $9.49 \times 10^{-12} \mathrm{e}^{380 / \mathrm{T}}$ & Zabarnick et al. [1988] \\
\hline (R62) & ${ }^{1} \mathrm{CH}_{2}+\mathrm{H} \rightarrow \mathrm{CH}+\mathrm{H}_{2}$ & $2.0 \times 10^{-10}$ & Moses et al. [2000] \\
\hline (R63) & $\begin{aligned}{ }^{1} \mathrm{CH}_{2}+\mathrm{H}_{2} & \rightarrow{ }^{3} \mathrm{CH}_{2}+\mathrm{H}_{2} \\
& \rightarrow \mathrm{CH}_{3}+\mathrm{H}\end{aligned}$ & $\begin{array}{l}1.26 \times 10^{-11} \\
9.24 \times 10^{-11}\end{array}$ & $\begin{array}{l}\text { Braun et al. }[1970], \\
\quad \text { Langford et al. }[1983]\end{array}$ \\
\hline (R64) & ${ }^{1} \mathrm{CH}_{2}+\mathrm{N}_{2} \rightarrow{ }^{3} \mathrm{CH}_{2}+\mathrm{N}_{2}$ & $5.1 \times 10^{-13} \mathrm{~T}^{0.5}$ & Ashfold et al. [1981] \\
\hline (R65) & ${ }^{3} \mathrm{CH}_{2}+\mathrm{CO}_{2} \rightarrow \mathrm{CH}_{2} \mathrm{O}+\mathrm{CO}$ & $1.40 \times 10^{-14}$ & Darwin and Moore [1995] \\
\hline (R66) & ${ }^{3} \mathrm{CH}_{2}+\mathrm{O}_{2} \rightarrow \mathrm{CO}+\mathrm{H}_{2} \mathrm{O}$ & $4.00 \times 10^{-13}$ & Tsang and Hampson [1986] \\
\hline (R67) & ${ }^{3} \mathrm{CH}_{2}+\mathrm{H} \rightarrow \mathrm{CH}+\mathrm{H}_{2}$ & $5.18 \times 10^{-11} \mathrm{e}^{675 / \mathrm{T}}$ & Devriendt et al. $[1995]$ \\
\hline (R68) & $\mathrm{CH}_{3}+\mathrm{O} \rightarrow \mathrm{CH}_{2} \mathrm{O}+\mathrm{H}$ & $1.40 \times 10^{-10}$ & Baulch et al. [1992] \\
\hline (R69) & $\mathrm{CH}_{3}+\mathrm{O}_{3} \rightarrow$ products & $5.40 \times 10^{-12} \mathrm{e}^{-220 / \mathrm{T}}$ & DeMore et al. [1997] \\
\hline (R70) & $\mathrm{CH}_{3}+\mathrm{H}+\mathrm{M} \rightarrow \mathrm{CH}_{4}+\mathrm{M}$ & $1.71 \times 10^{-24} \mathrm{~T}^{-1.80}$ & Baulch et al. [1994] \\
\hline (R71) & $\mathrm{CH}_{3}+\mathrm{OH} \rightarrow \mathrm{CH}_{2} \mathrm{OH}+\mathrm{H}$ & $1.31 \times 10^{-11}$ & Fagerstrom et al. [1994] \\
\hline & $\rightarrow \mathrm{CH}_{3} \mathrm{O}+\mathrm{H}$ & $1.60 \times 10^{-10}$ & Fagerstrom et al. [1994] \\
\hline (R72) & $\mathrm{CH}_{3}+\mathrm{OH}+\mathrm{M} \rightarrow \mathrm{CH}_{3} \mathrm{OH}+\mathrm{M}$ & $6.26 \times 10^{-18} \mathrm{~T}^{-3.8}$ & Fagerstrom et al. [1994] \\
\hline (R73) & $\mathrm{CH}_{3}+\mathrm{HO}_{2} \rightarrow \mathrm{CH}_{4}+\mathrm{O}_{2}$ & $5.99 \times 10^{-12}$ & Tsang and Hampson [1986] \\
\hline & $\rightarrow \mathrm{CH}_{3} \mathrm{O}+\mathrm{OH}$ & $3.30 \times 10^{-11}$ & Tsang and Hampson [1986] \\
\hline (R74) & $\mathrm{CH}_{3}+\mathrm{H}_{2} \mathrm{O}_{2} \rightarrow \mathrm{CH}_{4}+\mathrm{HO}_{2}$ & $2.00 \times 10^{-14} \mathrm{e}^{-300 / \mathrm{T}}$ & Tsang and Hampson [1986] \\
\hline (R75) & $\mathrm{CH}_{3}+\mathrm{CH}_{3} \rightarrow \mathrm{C}_{2} \mathrm{H}_{6}$ & $3.51 \times 10^{-7} \mathrm{~T}^{-7} \mathrm{e}^{-1390 / \mathrm{T}}$ & Baulch et al. [1994] \\
\hline (R76) & $\mathrm{CH}_{4}+\mathrm{O} \rightarrow \mathrm{CH}_{3}+\mathrm{OH}$ & $1.15 \times 10^{-15} \mathrm{~T}^{1.56} \mathrm{e}^{-4270 / \mathrm{T}}$ & Baulch et al. [1992] \\
\hline (R77) & $\mathrm{CH}_{4}+\mathrm{O}\left({ }^{1} \mathrm{D}\right) \rightarrow \mathrm{CH}_{3}+\mathrm{OH}$ & $1.13 \times 10^{-10}$ & DeMore et al. [1997] \\
\hline & $\rightarrow \mathrm{CH}_{2} \mathrm{O}+\mathrm{H}_{2}$ & $7.51 \times 10^{-12}$ & DeMore et al. [1997] \\
\hline (R78) & $\mathrm{CH}_{4}+\mathrm{OH} \rightarrow \mathrm{CH}_{3}+\mathrm{H}_{2} \mathrm{O}$ & $9.64 \times 10^{-13} \mathrm{e}^{-1418 / \mathrm{T}}$ & Sharkey and Smith [1993] \\
\hline (R79) & $\mathrm{CH}_{4}+\mathrm{CH} \rightarrow \mathrm{C}_{2} \mathrm{H}_{4}+\mathrm{H}$ & $2.49 \times 10^{-12}$ & Braun et al. [1967] \\
\hline (R80) & $\mathrm{HCO}+\mathrm{O} \rightarrow \mathrm{OH}+\mathrm{CO}$ & $5 \times 10^{-11}$ & Baulch et al. [1992] \\
\hline & $\rightarrow \mathrm{CO}_{2}+\mathrm{H}$ & $5 \times 10^{-11}$ & Baulch et al. [1992] \\
\hline (R81) & $\mathrm{HCO}+\mathrm{O}_{2} \rightarrow \mathrm{CO}+\mathrm{HO}_{2}$ & $2.19 \times 10^{-12} \mathrm{e}^{170 / \mathrm{T}}$ & Nesbitt et al. [1999] \\
\hline (R82) & $\mathrm{HCO}+\mathrm{H} \rightarrow \mathrm{CO}+\mathrm{H}_{2}$ & $1.5 \times 10^{-10}$ & Baulch et al. [1992] \\
\hline (R83) & $\mathrm{HCO}+\mathrm{HO}_{2} \rightarrow \mathrm{CH}_{2} \mathrm{O}+\mathrm{O}_{2}$ & $5.0 \times 10^{-11}$ & Tsang and Hampson [1986] \\
\hline (R84) & $\mathrm{CH}_{2} \mathrm{O}+\mathrm{O} \rightarrow \mathrm{HCO}+\mathrm{OH}$ & $6.85 \times 10^{-13} \mathrm{~T}^{0.57} \mathrm{e}^{-1390 / \mathrm{T}}$ & Baulch et al. [1992] \\
\hline (R85) & $\mathrm{CH}_{2} \mathrm{O}+\mathrm{H} \rightarrow \mathrm{HCO}+\mathrm{H}_{2}$ & $2.1 \times 10^{-16} \mathrm{~T}^{1.62} \mathrm{e}^{-1090 / \mathrm{T}}$ & Baulch et al. [1994] \\
\hline (R86) & $\mathrm{CH}_{2} \mathrm{O}+\mathrm{OH} \rightarrow \mathrm{HCO}+\mathrm{H}_{2} \mathrm{O}$ & $1.00 \times 10^{-11}$ & DeMore et al. [1997] \\
\hline (R87) & $\mathrm{CH}_{2} \mathrm{OH}+\mathrm{O} \rightarrow \mathrm{CH}_{2} \mathrm{O}+\mathrm{OH}$ & $1.50 \times 10^{-10}$ & Grotheer et al. [1989] \\
\hline (R87) & $\mathrm{CH}_{2} \mathrm{OH}+\mathrm{O}_{2} \rightarrow \mathrm{CH}_{2} \mathrm{O}+\mathrm{HO}_{2}$ & $9.1 \times 10^{-12}$ & DeMore et al. [1997] \\
\hline (R89) & $\mathrm{CH}_{2} \mathrm{OH}+\mathrm{H} \rightarrow \mathrm{CH}_{3}+\mathrm{OH}$ & $1.60 \times 10^{-10}$ & Tsang [1987] \\
\hline & $\rightarrow \mathrm{CH}_{2} \mathrm{O}+\mathrm{H}_{2}$ & $1.00 \times 10^{-11}$ & Tsang [1987] \\
\hline (R90) & $\mathrm{CH}_{3} \mathrm{O}+\mathrm{O} \rightarrow \mathrm{CH}_{2} \mathrm{O}+\mathrm{OH}$ & $1.00 \times 10^{-11}$ & Tsang and Hampson [1986] \\
\hline & $\rightarrow \mathrm{CH}_{3}+\mathrm{O}_{2}$ & $2.51 \times 10^{-11}$ & Zellner [1987] \\
\hline (R91) & $\mathrm{CH}_{3} \mathrm{O}+\mathrm{O}_{2} \rightarrow \mathrm{CH}_{2} \mathrm{O}+\mathrm{HO}_{2}$ & $3.90 \times 10^{-14} \mathrm{e}^{-900 / \mathrm{T}}$ & DeMore et al. [1997] \\
\hline (R92) & $\mathrm{CH}_{3} \mathrm{O}+\mathrm{H} \rightarrow \mathrm{CH}_{2} \mathrm{O}+\mathrm{H}_{2}$ & $3.01 \times 10^{-11}$ & Baulch et al. [1992] \\
\hline (R93) & $\mathrm{CH}_{3} \mathrm{O}+\mathrm{HO}_{2} \rightarrow \mathrm{CH}_{2} \mathrm{O}+\mathrm{H}_{2} \mathrm{O}_{2}$ & $5.0 \times 10^{-13}$ & Tsang [1987] \\
\hline (R94) & $\mathrm{CH}_{3} \mathrm{OH}+\mathrm{OH} \rightarrow \mathrm{CH}_{2} \mathrm{OH}+\mathrm{H}_{2} \mathrm{O}$ & $2.39 \times 10^{-18} \mathrm{~T}^{2} \mathrm{e}^{423 / \mathrm{T}}$ & Li and Williams [1996] \\
\hline & $\rightarrow \mathrm{CH}_{3} \mathrm{O}+\mathrm{H}_{2} \mathrm{O}$ & $1.66 \times 10^{-11} \mathrm{e}^{-854 / \mathrm{T}}$ & Warnatz [1984] \\
\hline (R95) & $\mathrm{C}_{2} \mathrm{H}_{2}+\mathrm{O} \rightarrow$ products & $3.01 \times 10^{-11} \mathrm{e}^{-1600 / \mathrm{T}}$ & DeMore et al. [1997] \\
\hline
\end{tabular}


Table 4. (continued)

\begin{tabular}{llll}
\hline & Reaction & \multicolumn{1}{c}{ Rate Coefficient ${ }^{\mathrm{a}}$} & \multicolumn{1}{c}{ Reference } \\
\hline (R96) & $\mathrm{C}_{2} \mathrm{H}_{2}+\mathrm{OH} \rightarrow$ products & $1.91 \times 10^{-12} \mathrm{e}^{-233 / \mathrm{T}}$ & Atkinson [1986] \\
(R97) & $\mathrm{C}_{2} \mathrm{H}_{4}+\mathrm{O} \rightarrow \mathrm{CH}_{2} \mathrm{O}+{ }^{3} \mathrm{CH}_{2}$ & $8.30 \times 10^{-12} \mathrm{e}^{-754 / \mathrm{T}}$ & Westenberg and DeHaas [1969] \\
(R98) & $\mathrm{C}_{2} \mathrm{H}_{4}+\mathrm{OH} \rightarrow$ products & $2.14 \times 10^{-12} \mathrm{e}^{411 / \mathrm{T}}$ & Atkinson [1986] \\
(R99) & $\mathrm{C}_{2} \mathrm{H}_{6}+\mathrm{OH} \rightarrow$ products & $8.60 \times 10^{-12} \mathrm{e}^{-1070 / \mathrm{T}}$ & DeMore et al. [1997] \\
\hline
\end{tabular}

${ }^{\mathrm{a}}$ Units are $\mathrm{s}^{-1}$ for photolysis reactions, $\mathrm{cm}^{3} \mathrm{~s}^{-1}$ for two-body reactions, and $\mathrm{cm}^{6} \mathrm{~s}^{-1}$ for three-body reactions. Photolysis rate coefficients are given at $10 \mathrm{~km}$ and $200 \mathrm{~km}$.

process is very efficient and occurs all the way down to the surface. Mercapto radical quickly reacts with $\mathrm{O}_{3}$ to form HSO (R43), which upon reaction with $\mathrm{O}_{2}$ forms $\mathrm{SO}_{2}$ (R50). The subsequent chemistry then follows that of $\mathrm{SO}_{2}$. Because there is no fast reaction that recycles sulfur atoms back to $\mathrm{H}_{2} \mathrm{~S}$, the $\mathrm{H}_{2} \mathrm{~S}$ concentration decreases rapidly with altitude, and the sulfur atoms eventually combine with $\mathrm{O}_{2}$ to form $\mathrm{SO}_{2}$. Therefore the mixing ratio of $\mathrm{SO}_{2}$ increases slightly with altitude just above the surface (see Figure 2).

[11] It is also noted that the mixing ratio of atomic sulfur at high altitudes increases due to its smaller molecular weight relative to $\mathrm{CO}_{2}$. Unlike on Venus, carbonyl sulfide (OCS) does not get formed with $\mathrm{SO}_{2}$ and $\mathrm{H}_{2} \mathrm{~S}$ outgassing, because the major reaction pathway that leads to the OCS production, $\mathrm{HS}+\mathrm{CO} \rightarrow \mathrm{OCS}+\mathrm{H}$, is found to be extremely slow under the martian atmospheric conditions of low temperature, and also the abundance of HS is quite small.

[12] The photochemical lifetime of $\mathrm{SO}_{2}$ is about $1.4 \times$ $10^{7} \mathrm{~s}$ (180 days); for $\mathrm{H}_{2} \mathrm{~S}$ it is $7.9 \times 10^{5} \mathrm{~s}$ ( 9 days), and for $\mathrm{SO}$ it is $1.7 \times 10^{4} \mathrm{~s}(4.6$ hours $)$. In a relatively short time of an hour, ordinary convective processes alone can reduce the mixing ratios of outgassed species by factors of $10^{4}-10^{5}$ approximately $50 \mathrm{~km}$ from the source, and the time for spreading the source material more or less uniformly over the planet (with corresponding dilution factor of $\sim 10^{8}$ ) would be approximately one year (N. Renno, personal communication, 2003). In view of these time scales and the above photochemical lifetimes of the species, detection of any sulfur species would indicate presence of currently active hot spot(s) in close proximity of the observation.
Unless the source flux is extremely large, detection far from a transient source would be unlikely due to dilution.

\section{Hydrocarbon Chemistry}

[13] Methane has not been detected on Mars. Since the observations represent an average over large areas, methane, like the sulfur species (section 3), could go undetected even if there was copious outgassing from a localized source, especially a transient one. We use the photochemical model to calculate the abundances of new species that are formed upon the introduction of $\mathrm{CH}_{4}$ into the Martian atmosphere by outgassing. The new species included in the calculations are: $\mathrm{CH}$, singlet methylene $\left({ }^{1} \mathrm{CH}_{2}\right)$, triplet methylene $\left({ }^{3} \mathrm{CH}_{2}\right)$, methyl radical $\left(\mathrm{CH}_{3}\right), \mathrm{CH}_{4}, \mathrm{HCO}$, formaldehyde $\left(\mathrm{CH}_{2} \mathrm{O}\right), \mathrm{CH}_{2} \mathrm{OH}, \mathrm{CH}_{3} \mathrm{O}$, methyl alcohol $\left(\mathrm{CH}_{3} \mathrm{OH}\right), \mathrm{C}_{2} \mathrm{H}_{2}$, $\mathrm{C}_{2} \mathrm{H}_{4}$, and ethane $\left(\mathrm{C}_{2} \mathrm{H}_{6}\right)$. The list of important reactions is given in Table 4 . The principal reaction pathways are illustrated in Figure 3 and will be discussed later in this section.

[14] The global average upper limit of methane is 0.02 ppm [Maguire, 1997]. Starting with $0.02 \mathrm{ppm}$ of methane at the surface, we increase the concentration gradually in subsequent model runs up to $100 \mathrm{ppm}$ above any possible hot spots. Larger or smaller enhancement factors may apply over hot spots, as discussed in the context of the sulfur species (section 3). Table 5 tabulates a sample of the model results. For the purpose of illustration, the result of a "nominal" case, Model L, is summarized here. In this case, the surface concentration of methane is assumed to be

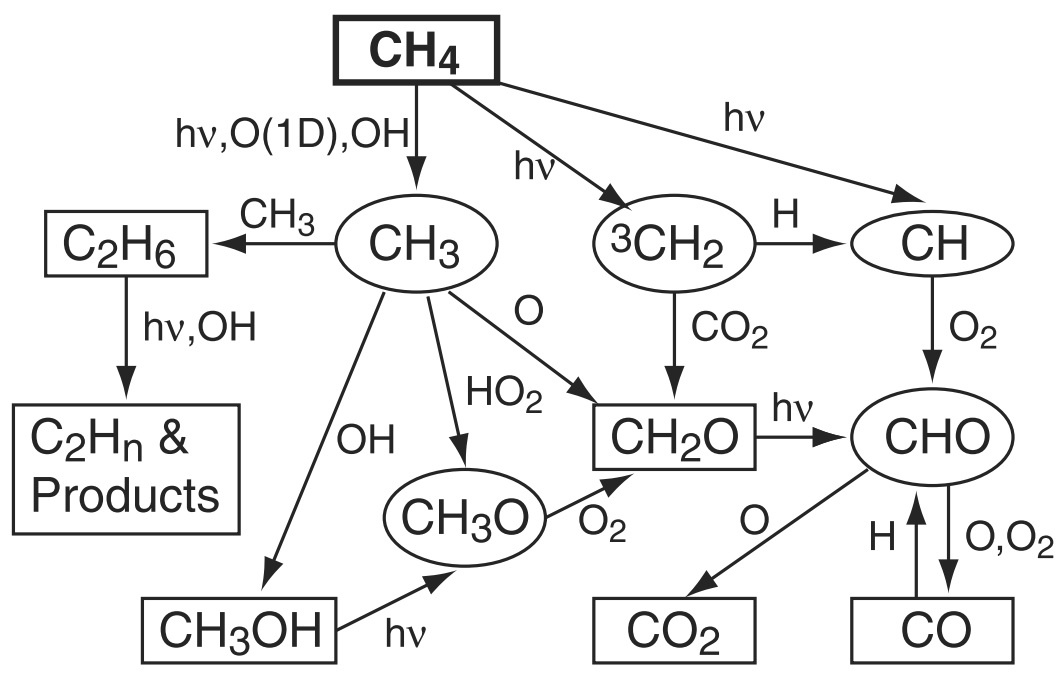

Figure 3. Schematic diagram illustrating the important reaction pathways of hydrocarbon chemistry in Martian atmosphere. Stable species are in rectangles, radicals are in ovals, and outgassing species are in bold. 
Table 5. Model Results for Methane Chemistry in the Martian Atmosphere ${ }^{\mathrm{a}}$

\begin{tabular}{|c|c|c|c|c|c|c|c|c|c|}
\hline \multirow[b]{3}{*}{ Model } & \multirow{3}{*}{$\frac{\text { Outgassing }}{\frac{\mathrm{CH}_{4}}{\text { Surface Mixing }}}$} & \multicolumn{8}{|c|}{ Major Photochemical Products } \\
\hline & & \multicolumn{2}{|c|}{$\mathrm{CH}_{4}$} & \multicolumn{2}{|c|}{$\mathrm{CH}_{2} \mathrm{O}$} & \multicolumn{2}{|c|}{$\mathrm{CH}_{3} \mathrm{OH}$} & \multicolumn{2}{|c|}{$\mathrm{C}_{2} \mathrm{H}_{6}$} \\
\hline & & Mixing & Column & Mixing & Column & Mixing & Column & Mixing & Column \\
\hline $\mathrm{J}$ & $2 \times 10^{-8}$ & $2 \times 10^{-8}$ & $2.0 \times 10^{15}$ & $6.0 \times 10^{-15}$ & $4.0 \times 10^{9}$ & $1.4 \times 10^{-15}$ & $2.1 \times 10^{8}$ & $2.1 \times 10^{-18}$ & $2.3 \times 10^{5}$ \\
\hline $\mathrm{K}$ & $1 \times 10^{-6}$ & $1 \times 10^{-6}$ & $1.0 \times 10^{17}$ & $2.9 \times 10^{-13}$ & $2.0 \times 10^{11}$ & $4.3 \times 10^{-14}$ & $8.1 \times 10^{9}$ & $6.4 \times 10^{-16}$ & $9.7 \times 10^{7}$ \\
\hline $\mathrm{L}$ & $1 \times 10^{-4}$ & $1 \times 10^{-4}$ & $1.0 \times 10^{19}$ & $2.8 \times 10^{-11}$ & $1.6 \times 10^{13}$ & $4.3 \times 10^{-12}$ & $8.2 \times 10^{11}$ & $6.4 \times 10^{-12}$ & $9.6 \times 10^{11}$ \\
\hline \multicolumn{2}{|c|}{ Photochemical lifetime } & \multicolumn{2}{|c|}{$2.1 \times 10^{10} \mathrm{~s}(670$ years $)$} & \multicolumn{2}{|c|}{$2.7 \times 10^{4} \mathrm{~s}(7.5$ hours $)$} & \multicolumn{2}{|c|}{$6.4 \times 10^{6} \mathrm{~s}$ (74 days) } & \multicolumn{2}{|c|}{$7.9 \times 10^{8} \mathrm{~s}(25$ years $)$} \\
\hline
\end{tabular}

"Mixing ratios (labeled "mixing") and the column abundances (in $\mathrm{cm}^{-2}$, labeled "column") are compared at $10 \mathrm{~km}$ in this listing. Lifetime of a species indicates the time to destroy all of that species in the atmosphere photochemically.

$100 \mathrm{ppm}$. The most abundant hydrocarbon species are $\mathrm{CH}_{4}$, $\mathrm{CH}_{2} \mathrm{O}, \mathrm{CH}_{3} \mathrm{OH}$, and $\mathrm{C}_{2} \mathrm{H}_{6}$, and the calculated mixing ratios for these species at $10 \mathrm{~km}$ are $1 \times 10^{-4}, 2.8 \times 10^{-11}, 4.3 \times$ $10^{-12}$, and $6.4 \times 10^{-12}$, respectively (see Table 5). The number densities and mixing ratios of important species of hydrocarbon chemistry are plotted in Figure 5 for this case.

[15] At high altitudes above $80 \mathrm{~km}$, methane is photodissociated into $\mathrm{CH},{ }^{3} \mathrm{CH}_{2},{ }^{1} \mathrm{CH}_{2}$, and $\mathrm{CH}_{3}$ (R52). ${ }^{3} \mathrm{CH}_{2}$ reacts with the background gas $\mathrm{CO}_{2}$ to form $\mathrm{CH}_{2} \mathrm{O}$ (R65). This explains the upper peak in $\mathrm{CH}_{2} \mathrm{O}$ concentration at high altitudes. At low altitudes, the oxidation of methane by $\mathrm{O}\left({ }^{1} \mathrm{D}\right)$ and $\mathrm{OH}$ forms $\mathrm{CH}_{3}$ (R76), (R77). $\mathrm{CH}_{3}$ reacts with $\mathrm{O}$ to form $\mathrm{CH}_{2} \mathrm{O}$ (R68) and with $\mathrm{OH}$ to form $\mathrm{CH}_{3} \mathrm{OH}$ (R71). $\mathrm{C}_{2} \mathrm{H}_{6}$ is produced by recombination of $\mathrm{CH}_{3}$ (R74) and its concentration is proportional to the square of that of $\mathrm{CH}_{3}$. From $\mathrm{C}_{2} \mathrm{H}_{6}$, other hydrocarbons with two or more carbon atoms can be formed. The model also shows that the increase in the concentrations of $\mathrm{CH}_{2} \mathrm{O}$ and other single carbon species is proportional to the increase in the $\mathrm{CH}_{4}$ abundance, as would be expected, whereas increase in the concentrations of $\mathrm{C}_{2} \mathrm{H}_{6}$ and other double carbon species is proportional to square of the increase in the $\mathrm{CH}_{4}$ abundance (see Table 5).

[16] We find that the abundances of hydrocarbons derived from the methane photochemistry are very low even when the concentration of methane is relatively large in the atmosphere. The reason is that the dissociation of methane, the starting point of the hydrocarbon chemistry, is very inefficient in the Martian atmosphere. The destruction rates of methane via different channels are shown and compared in Figure 4. Photolysis of methane occurs only high in the atmosphere, at around $90 \mathrm{~km}$ (see Figure 4), because UV destruction of methane in the lower atmosphere is largely shielded by the much more abundant $\mathrm{CO}_{2}$ which has an overlapping absorption cross-section spectrum with methane. In the lower atmosphere the fastest pathway to break down methane is by reaction with $\mathrm{OH}$ (R77), the photoproduct of water. However, increasing the amount of water does not significantly promote the destruction of methane. The reason is that once formed, $\mathrm{OH}$ is quickly removed by $\mathrm{CO}$ and $\mathrm{O}$ via reactions R16 and R17. Compared to R16 and $\mathrm{R} 17$, the reaction rate of $\mathrm{R} 77$ is much slower.

[17] The photochemical lifetime of methane is found to be about 670 years, which is the longest amongst the principal hydrocarbon species (see Table 5). This fact, combined with the larger abundance of methane, means that methane may be the easiest hydrocarbon to detect even far from the source region, provided that its source flux is reasonably large. On the other hand, formaldehyde has a very short lifetime of only several hours. Therefore if formaldehyde is detected, it would imply a nearly continuous supply of very large quantities of methane, whose oxidation forms $\mathrm{CH}_{2} \mathrm{O}$. How-

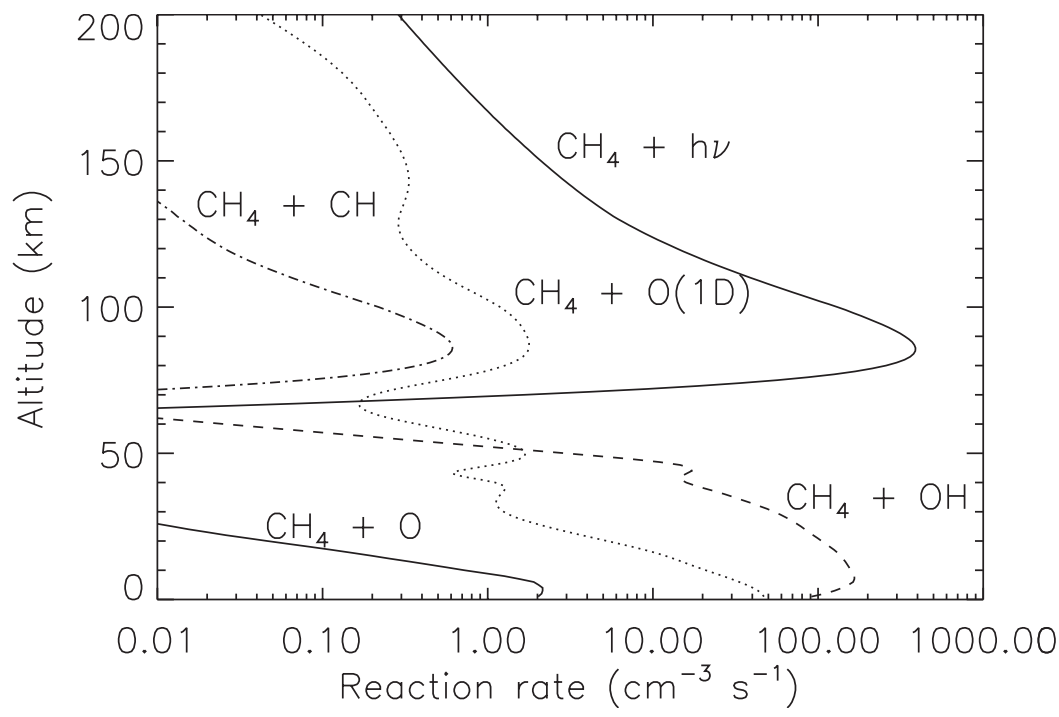

Figure 4. Destruction rates of methane versus altitude, assuming $100 \mathrm{ppm}$ of methane is outgassed at the surface of Mars. 

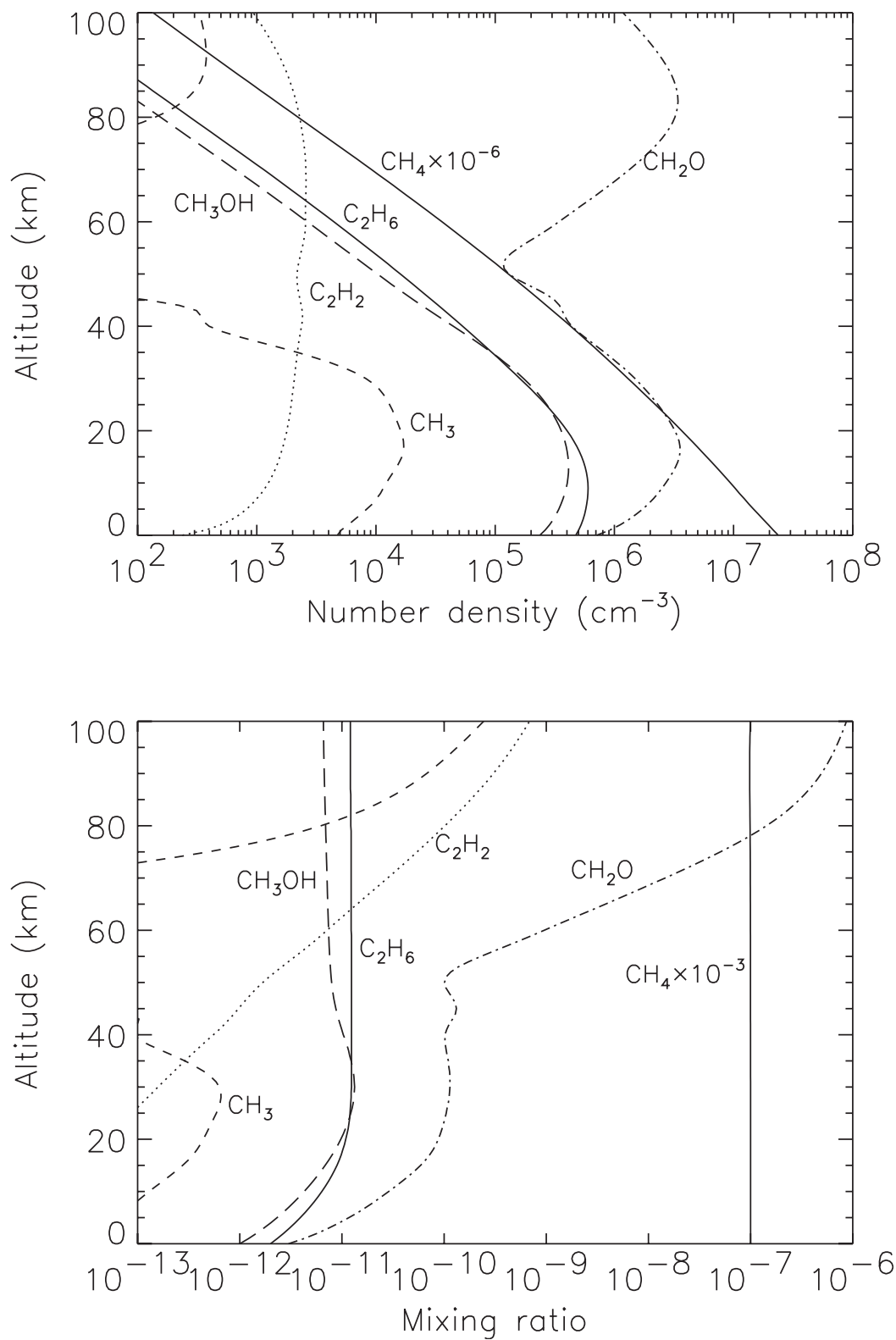

Figure 5. Number densities and mixing ratios versus altitude for important species calculated with $100 \mathrm{ppm}$ of $\mathrm{CH}_{4}$ at the surface of Mars (Model L).

ever, if formaldehyde is detected without any methane associated with it, there maybe other possibilities for its existence. For example, formaldehyde itself might have been outgassing directly, or it maybe formed in surface reactions between condensed phase $\mathrm{H}_{2} \mathrm{O}$ and $\mathrm{CO}_{2}$ under the action of solar ultraviolet radiation.

\section{Location Consideration}

[18] In the past the most active outgassing presumably occurred in the vast Tharsis volcano region. Could it be that small vents in the calderas of the volcanoes are still sending small quantities of gases into the Martian atmosphere? It would be desirable to investigate the Tharsis volcano region in future observations. Another area would be the Martian high latitudes where most of the signs of recent water seepage and ground water runoff have been seen. The calculations presented in this paper assume that outgassing is taking place from the surface of Mars. Should there be vents in the calderas of the volcanoes, the source of outgassing would move up to higher elevations. This would have an impact on the calculated abundance of the photochemical products of outgassed species once they react with the known Martian atmosphere. This is due to a number of factors including the condensation of water vapor between 10 and $20 \mathrm{~km}$ and the varying distribution of oxygen bearing species with altitudes. For example, if the source of outgassing lies at $10 \mathrm{~km}$ above the surface of Mars, the column abundances (in $\mathrm{cm}^{-2}$ ) at $10 \mathrm{~km}$ of the most abundant constituents, assuming 100 ppm each of $\mathrm{SO}_{2}$, $\mathrm{H}_{2} \mathrm{~S}$, and $\mathrm{CH}_{4}$ would be $\mathrm{SO}_{2}=1.6 \times 10^{19}, \mathrm{H}_{2} \mathrm{~S}=4.0 \times$ $10^{18}, \mathrm{SO}=1.4 \times 10^{17} ; \mathrm{CH}_{4}=1.0 \times 10^{19}, \mathrm{CH}_{2} \mathrm{O}=1.6 \times$ $10^{13}, \mathrm{CH}_{3} \mathrm{OH}=4.3 \times 10^{11}, \mathrm{C}_{2} \mathrm{H}_{6}=3.2 \times 10^{11}$. If the source with 100 ppm each of $\mathrm{SO}_{2}, \mathrm{H}_{2} \mathrm{~S}$, and $\mathrm{CH}_{4}$ is situated 
on top of Olympus Mons (27 km above reference level), then the column abundances (in $\mathrm{cm}^{-2}$ ) at $27 \mathrm{~km}$ would be $\mathrm{SO}_{2}=2.6 \times 10^{18}, \mathrm{H}_{2} \mathrm{~S}=6.8 \times 10^{17}, \mathrm{SO}=1.4 \times 10^{17}$; $\mathrm{CH}_{4}=1.3 \times 10^{18}, \mathrm{CH}_{2} \mathrm{O}=1.1 \times 10^{13}, \mathrm{CH}_{3} \mathrm{OH}=1.8 \times$ $10^{10}, \mathrm{C}_{2} \mathrm{H}_{6}=1.3 \times 10^{9}$.

\section{Conclusion}

[19] In this paper we have shown that if outgassing is taking place somewhere on Mars today, only a handful of new species with significant abundance would be formed as a result of photochemical processes involving the products of outgassing. In particular the outgassed $\mathrm{H}_{2} \mathrm{~S}$ and $\mathrm{SO}_{2}$ would produce significant amounts of only SO. For example, $100 \mathrm{ppm}$ each of $\mathrm{H}_{2} \mathrm{~S}$ and $\mathrm{SO}_{2}$ at the surface would result in a column abundance of $7.8 \times 10^{16} \mathrm{~cm}^{-2}$ of SO. The bulk of $\mathrm{H}_{2} \mathrm{SO}_{4}$ produced in the photochemistry is expected to condense out. Similarly, photochemistry of any outgassed $\mathrm{CH}_{4}$ would produce appreciable amounts of only formaldehyde and methyl alcohol. With 100 ppm of outgassed $\mathrm{CH}_{4}$ at the surface, for example, $1.6 \times 10^{13} \mathrm{~cm}^{-2}$ of $\mathrm{CH}_{2} \mathrm{O}$ and $8.2 \times 10^{11} \mathrm{~cm}^{-2}$ of $\mathrm{CH}_{3} \mathrm{OH}$ would result. Unlike the sulfur species, the hydrocarbon photochemical products would be far less abundant. The abundance of any halogens outgassed from the hot spots is expected to be small; therefore the photochemical products would be even less abundant. Thus the best candidates for the chemical markers of any hot spots on Mars are $\mathrm{SO}_{2}, \mathrm{H}_{2} \mathrm{~S}, \mathrm{CH}_{4}$, possibly $\mathrm{SO}, \mathrm{CH}_{2} \mathrm{O}$, and $\mathrm{CH}_{3} \mathrm{OH}$. The largest source of methane in the Earth's atmosphere is from fermentation of the biomass, with smaller contributions from fossilized matter. Methane and its products could serve as possible biomarkers in the Martian atmosphere as well. To conclude, we are not suggesting that hot spots exist on Mars today, but if they did, abovementioned chemical species predicted by our photochemical model would be their best indicator. Finally, it should be stressed that the aim of the present study is to identify possible hot spot related species in the Martian atmosphere. Should chemical signatures of hot spots be detected and the species abundance measured in future observations of Mars, their interpretation would warrant a more sophisticated model that combines photochemistry with full dynamics including general circulation.

[20] Acknowledgments. SKA acknowledges support received from NASA's Planetary Atmospheres Program and NASA's Mars Program Office at JPL for U.S. participation in the Mars Express Project. We thank Scott Edgington and Eric Wilson for help with the model and Yuk Yung for helpful comments on the manuscript.

\section{References}

Ashfold, M. N. R., M. A. Fullstone, G. Hancock, and G. W. Ketley, Singlet methylene kinetics: Direct measurements of removal rates of $\tilde{a}^{1} \mathrm{~A}_{1}$ and $\tilde{\mathrm{b}}^{1} \mathrm{~B}_{1} \mathrm{CH}_{2}$ and $\mathrm{CD}_{2}$, Chem. Phys., 55, 245-257, 1981.

Atkinson, R., Kinetics and mechanisms of the gas-phase reactions of the hydroxyl radical with organic compounds under atmospheric conditions, Chem. Rev., 86, 69, 1986.

Atkinson, R., D. L. Baulch, R. A. Cox, R. F. Hampson Jr., J. A. Kerr, and J. Troe, Evaluated kinetic and photochemical data for atmospheric chemistry, J. Phys. Chem. Ref., 18, suppl. 3, 881-1097, 1989.

Atkinson, R., D. L. Baulch, R. A. Cox, R. F. Hampson Jr., J. A. Kerr, M. J. Rossi, and J. Troe, Evaluated kinetic and photochemical data for atmospheric chemistry: IUPAC subcommittee on gas kinetic data evaluation for atmospheric chemistry, J. Phys. Chem. Ref. Data, 26, suppl. VI, $1329-1499,1997$.
Atreya, S. K., and Z. Gu, Stability of the martian atmosphere: Is heterogeneous catalysis essential?, J. Geophys. Res., 99, 13,133-13,145, 1994. Balko, B. A., J. Zhang, and Y. T. Lee, Photodissociation of ethylene at 193 nm, J. Chem. Phys., 97, 935-942, 1992.

Baulch, D. L., et al., Evaluated kinetic data for combustion modeling, J. Phys. Chem. Ref. Data, 21, 411-429, 1992.

Baulch, D. L., et al., Evaluated kinetic data for combustion modeling, J. Phys. Chem. Ref. Data, 23, (suppl. I), 847-1033, 1994.

Braun, W., J. R. McNesby, and A. N. Bass, Flash photolysis of methane in the vacuum ultraviolet: II. Absolute rate constants for reactions of $\mathrm{CH}$ with methane, hydrogen, and nitrogen, J. Chem. Phys., 46, 2071, 1967.

Braun, W., A. M. Bass, and M. Pilling, Flash photolysis of ketene and diazomethane: The production and reaction kinetics of triplet and singlet methylene, J. Chem. Phys., 52, 5131-5143, 1970.

Bulatov, V. P., O. M. Sarkisov, and M. Kozliner, Mechanism of photooxidation of hydrogen sulfide in the atmosphere, Khim. Fiz., 5, 1031-1036, 1986.

Christensen, L. E., M. Okumura, S. P. Sander, R. J. Salawitch, G. C. Toon, B. Sen, J.-F. Blavier, and K. W. Jucks, Kinetics of $\mathrm{HO}_{2}+\mathrm{HO}_{2} \rightarrow \mathrm{H}_{2} \mathrm{O}_{2}+$ $\mathrm{O}_{2}$ : Implications for stratospheric $\mathrm{H}_{2} \mathrm{O}_{2}$, Geophys. Res. Lett., 29(9), 1299 , doi:10.1029/2001GL014525, 2002.

Chung, K., J. G. Calvert, and J. W. Bottenheim, The photochemistry of sulfur dioxide excited within its first allowed band $(3130 \AA)$ and the "forbidden" band (3700-4000 ̊), Int. J. Chem. Kinet., 7, 161, 1975.

Darwin, D. C., and C. B. Moore, Reaction-rate constant $(295 \mathrm{~K})$ for ${ }^{3} \mathrm{CH}_{2}$ with $\mathrm{H}_{2} \mathrm{~S}, \mathrm{SO}_{2}$, and $\mathrm{NO}_{2}$ : Upper-bounds for rate constants with less reactive partners, J. Phys. Chem., 99, 13,467-13,470, 1995.

DeMore, W. B., D. M. Golden, R. F. Hampson, C. J. Howard, M. J. Kurylo, J. J. Margitan, M. J. Molina, A. R. Ravishankara, and R. T. Watson, Chemical kinetics and photochemical data for use in stratospheric modeling, evaluation 7, JPL Publ. 85-37, Jet Propulsion Lab., Pasadena, Calif., 1985.

DeMore, W. B., S. P. Sander, D. M. Golden, R. F. Hampson, M. J. Kurylo, C. J. Howard, A. R. Ravishankara, and M. J. Molina, Chemical kinetics and photochemical data for use in stratospheric modeling, JPL Publ. 8741, Jet Propulsion Lab., Pasadena, Calif., 1987.

DeMore, W. B., D. M. Golden, R. F. Hampson, C. J. Howard, M. J. Kurylo, M. J. Molina, A. R. Ravishankara, and S. P. Sander, Chemical kinetics and photochemical data for use in stratospheric modeling, evaluation 9, JPL Publ. 90-1, Jet Propulsion Lab., Pasadena, Calif., 1990.

DeMore, W. B., C. J. Howard, S. P. Sander, A. R. Ravishankara, D. M. Golden, C. E. Kolb, R. F. Hampson, M. J. Molina, and M. J. Kurylo, Chemical kinetics and photochemical data for use in stratospheric modeling, JPL Publ. 92-20, Jet Propulsion Lab., Pasadena, Calif., 1992.

DeMore, W. B., S. P. Sander, D. M. Golden, R. F. Hampson, M. J. Kurylo, C. J. Howard, A. R. Ravishankara, C. E. Kolb, and M. J. Molina, Chemical kinetics and photochemical data for use in stratospheric modeling, evaluation 12, JPL Publ. 97-4, Jet Propulsion Lab., Pasadena, Calif., 1997.

Devriendt, K., M. Van Poppel, W. Boullart, and J. Peeters, Kinetic investigation of the $\mathrm{CH}_{2}\left(\mathrm{X}^{3} \mathrm{~B}_{1}\right)+\mathrm{H} \rightarrow \mathrm{CH}\left(\mathrm{X}^{2} \mathrm{II}\right)+\mathrm{H}_{2}$ reaction in the temperature range $400 \mathrm{~K}<\mathrm{T}<1000 \mathrm{~K}$, J. Phys. Chem., 99, 16,953-16,959, 1995.

Fagerstrom, K., A. Lund, G. Mahmoud, J. T. Jodkowski, and E. Ratajczak, Pressure and temperature dependence of the gas-phase reaction between methyl and hydroxyl radicals, Chem. Phys. Lett., 224, 43-50, 1994.

Farquhar, J., J. Savarino, T. L. Jackson, and M. H. Thiemens, Evidence of atmospheric sulphur in the martian regolith from sulphur isotopes in meteorites, Nature, 404, 50, 2000.

Fulle, D., and H. Hippler, The temperature and pressure dependence of the reaction $\mathrm{CH}+\mathrm{H}_{2} \leftrightarrow \mathrm{CH}_{3} \leftrightarrow \mathrm{CH}_{2}+\mathrm{H}$, J. Chem. Phys., 106, 8691-8698, 1997.

Gentieu, E. P., and J. E. Mentall, Formaldehyde absorption coefficients in the vacuum ultraviolet (650-1850 Angstroms), Science, 169, 681-683, 1970.

Gericke, K. H., and F. J. Comes, Energy partitioning in the reaction $\mathrm{O}\left({ }^{1} \mathrm{D}\right)+$ $\mathrm{H}_{2} \mathrm{O} \rightarrow \mathrm{OH}+\mathrm{OH}$ : The influence of $\mathrm{O}\left({ }^{1} \mathrm{D}\right)$ translational energy on the reaction rate constant, Chem. Phys. Lett., 81, 218-221, 1981.

Gladstone, G. R., M. Allen, and Y. L. Yung, Hydrocarbon photochemistry in the upper atmosphere of Jupiter, Icarus, 119, 1-52, 1996.

Glicker, S., and L. J. Stief, Photolysis of formaldehyde at 1470 and $1236 \AA$, J. Chem. Phys., 54, 2852-2857, 1971.

Grotheer, H., G. Riekert, D. Walter, and T. Just, Reactions of hydroxymethyl and hydroxyethyl radicals with molecular and atomic oxygen, Symp. Int. Combust. Proc., 22, 963, 1989.

Hagége, J., P. C. Roberge, and C. Vermeil, Methanol photochemistry: Collision-induced phenomena, Ber. Bunsenges. Phys. Chem., 72, 138-141, 1968.

Heck, A. J. R., R. N. Zare, and D. W. Chandler, Photofragment imaging of methane, J. Chem. Phys., 104, 4019-4030, 1996. 
Hochanadel, C. J., T. J. Sworski, and P. J. Ogren, Ultraviolet spectrum and reaction kinetics of the formyl radical, J. Phys. Chem., 84, 231-235, 1980.

König, R., S. Mory, A. Rosenfeld, J. Ladermann, R. Grundwald, and G. Winkelmann, Laser absorption and fluorescence spectroscopy of excited molecules in nanosecond time scale, J. Mol. Struct., 114, 101-104, 1984.

Korablev, O. I., M. Acherman, V. A. Krasnopolsky, V. I. Moroz, C. Muller, A. V. Rodin, and S. K. Atreya, Tentative identification of formaldehyde in the martian atmosphere, Planet. Space Sci., 41, 441-451, 1993.

Krasnopolsky, V. A., Photochemistry of the martian atmosphere (mean conditions), Icarus, 101, 313-332, 1993.

Krasnopolsky, V. A., G. L. Bjoraker, M. J. Mumma, and D. E. Jennings, High-resolution spectroscopy of Mars at 3.7 and $8 \mu \mathrm{m}$ : A sensitive search for $\mathrm{H}_{2} \mathrm{O}_{2}, \mathrm{H}_{2} \mathrm{CO}, \mathrm{HCl}$, and $\mathrm{CH}_{4}$, and detection of $\mathrm{HDO}, J$. Geophys. Res., 102, 6526-6534, 1997 .

Langford, A. O., H. Petek, and C. B. Moore, Collisional removal of $\mathrm{CH}_{2}\left({ }^{1} \mathrm{~A}_{1}\right)$-Absolute rate constants for atomic and molecular collisional partners at $291 \mathrm{~K}, J$. Chem. Phys., 78, 6650-6659, 1983.

Larson, C. W., P. H. Stewart, and D. M. Golden, Pressure and temperature dependence of reactions proceeding via a bound complex: An approach for combustion and atmospheric chemistry modelers, Int. J. Chem. Kinet., 20, 27-40, 1988

Lewis, B. R., and J. H. Carver, Temperature dependence of the carbon dioxide photoabsorption cross section between 1200 and $1970 \AA$, J. Quant. Spectrosc. Radiat. Transfer, 30, 297-309, 1983.

Li, S. C., and F. A. Williams, Experimental and numerical studies of twostage methanol flames, Symp. Int. Combust. Proc., 26, 1017-1024, 1996.

Maguire, W. S., Martian isotopic ratios and upper limits for possible minor constituents as derived from Mariner 9 infrared spectrometer data, Icarus, 32, 85-97, 1977

Malin, M. C., and K. S. Edgett, Evidence for recent groundwater seepage and surface runoff on Mars, Science, 288, 2330-2335, 2000.

Mentall, J. E., E. P. Gentieu, M. Krauss, and D. Neumann, Photoionization and absorption spectrum of formaldehyde in the vacuum ultraviolet, J. Chem. Phys., 55, 5471-5479, 1971.

Mordaunt, D. H., I. R. Lambert, G. P. Morley, M. N. R. Ashford, R. N. Dixon, and C. M. Western, Primary product channels in the photodissociation of methane at $121.6 \mathrm{~nm}, J$. Chem. Phys., 98, 2054-2065, 1993.

Moses, J. I., B. Bézard, E. Lellouch, G. R. Gladstone, H. Feuchtgruber, and M. Allen, Photochemistry of Saturn's atmosphere, I. Hydrocarbon chemistry and comparisons with ISO observations, Icarus, 143, 244-298, 2000.

Nair, H., M. Allen, A. D. Anbar, Y. L. Yung, and R. T. Clancy, A photochemical model of the martian atmosphere, Icarus, 111, 124-150, 1994. Nesbitt, F. L., J. F. Gleason, and L. J. Stief, Temperature dependence of the rate constant for the reaction $\mathrm{HCO}+\mathrm{O}_{2} \rightarrow \mathrm{HO}_{2}+\mathrm{CO}$ at T $=200-398 \mathrm{~K}$, J. Phys. Chem., Ser. A, 103, 3038-3043, 1999.

Nicholas, J. E., C. A. Amodio, and M. J. Baker, Kinetics and mechanism of the decomposition of $\mathrm{H}_{2} \mathrm{~S}, \mathrm{CH}_{3} \mathrm{SH}$, and $\left(\mathrm{CH}_{3}\right)_{2} \mathrm{~S}$ in a radio-frequency pulse discharge, J. Chem. Soc. Faraday Trans. 1, 75, 1868, 1979.

Okabe, H., Fluorescence and predissociation of sulfur dioxide, J. Am. Chem. Soc., 93, 7095-7096, 1971.

Okabe, H., Photochemistry of Small Molecules, John Wiley, New York, 1978.

Person, J. C., and P. P. Nicole, Isotope effects in the photoionization yields and in the absorption cross sections for methanol, ethanol, methyl bromide, and ethyl bromide, J. Chem. Phys., 55, 3390-3397, 1971.

Phillips, L. F., Absolute absorption cross sections for SO between $190 \mathrm{~nm}$ and $235 \mathrm{~nm}, J$. Phys. Chem., 85, 3994-4000, 1981.

Reiner, T., and F. Arnold, Laboratory investigations of gaseous sulfuric acid formation via $\mathrm{SO}_{3}+\mathrm{H}_{2} \mathrm{O}+M \rightarrow \mathrm{H}_{2} \mathrm{SO}_{4}+M$ : Measurement of the rate constant and product identification, J. Chem. Phys., 101, 7399-7407, 1994.
Satyapal, S., and R. Bersohn, Photodissociation of acetylene at $193.3 \mathrm{~nm}$, J. Phys. Chem., 95, 8004-8006, 1991.

Sharkey, P., and I. W. M. Smith, Kinetics of elementary reactions at low temperatures: Rate constants for the reactions of $\mathrm{OH}$ with $\mathrm{HCl}(298 \geq$ $\mathrm{T} / \mathrm{K} \geq 138), \mathrm{CH}_{4}(298 \geq \mathrm{T} / \mathrm{K} \geq 178)$ and $\mathrm{C} 2 \mathrm{H} 6(298 \geq \mathrm{T} / \mathrm{K} \geq 138)$, J. Chem. Soc. Faraday Trans., 89, 631-638, 1993.

Shemansky, D. E., $\mathrm{CO}_{2}$ extinction coefficient 1700-3000 А, J. Chem. Phys., 56, 1582-1587, 1972.

Singleton, D. L., and R. J. Cvetanovic, Evaluated chemical kinetic data for the reactions of atomic oxygen $\mathrm{O}\left({ }^{3} \mathrm{P}\right)$ with sulfur containing compounds, J. Phys. Chem. Ref. Data, 17, 1377, 1988.

Stachnik, R. A., and M. J. Molina, Kinetics of the reactions of SH radicals with $\mathrm{NO}_{2}$ and $\mathrm{O}_{2}$, J. Phys. Chem., 91, 4603, 1987.

Stull, D. R., Vapor pressure of pure substances-Inorganic compounds, Ind. Eng. Chem., 39, 540-550, 1947.

Summers, M. E., B. J. Lieb, E. Chapman, and Y. L. Yung, Atmospheric biomakers of subsurface life on Mars, Geophys. Res. Lett., 29(24), 2171, doi:10.1029/2002GL015377, 2002.

Suto, M., X. Wang, and L. C. Lee, Fluorescence from VUV excitation of formaldehyde, J. Chem. Phys., 85, 4228-4233, 1986.

Thompson, B. A., P. Harteck, and R. R. Reeves Jr., Ultraviolet absorption coefficients of $\mathrm{CO}_{2}, \mathrm{CO}, \mathrm{O}_{2}, \mathrm{H}_{2} \mathrm{O}, \mathrm{N}_{2} \mathrm{O}, \mathrm{NH}_{3}, \mathrm{NO}, \mathrm{SO}_{2}$, and $\mathrm{CH}_{4}$ between 1850 and $4000 \AA$ A, J. Geophys. Res., 68, 6431-6436, 1963.

Tsang, W., Chemical kinetic data base for combustion chemistry: part 2, Methanol, J. Phys. Chem. Ref. Data, 16, 471, 1987.

Tsang, W., and R. F. Hampson, Chemical kinetic data base for combustion chemistry: part I. Methane and related compounds, J. Phys. Chem. Ref. Data, 15, 1087, 1986.

Walker, J. C. G., Evolution of the Atmosphere, 205 pp., Macmillan, Old Tappan, N. J., 1997.

Wang, N. S., and C. J. Howard, Kinetics of the reactions of HS and HSO with $\mathrm{O}_{3}$, J. Phys. Chem., 94, 8787, 1990.

Wänke, H., and G. Dreibus, Chemistry and accretion history of Mars, Phil. Trans. Phys. Sci. Eng., 349, 285-293, 1994.

Warnatz, J., Rate coefficients in the $\mathrm{C} / \mathrm{H} / \mathrm{O}$ system, in Combustion Chemistry, edited by W. C. Gardiner, p. 197, Springer-Verlag, New York, 1984.

Watanabe, K., E. C. Y. Inn, and M. Zelikoff, Absorption coefficients of oxygen in the vacuum ultraviolet, J. Chem. Phys., 21, 1026-1030, 1953.

Welge, K. H., Photolysis of $\mathrm{O}_{\mathrm{x}}, \mathrm{HO}_{\mathrm{x}}, \mathrm{CO}_{\mathrm{x}}$ and $\mathrm{SO}_{\mathrm{x}}$ compounds, Can. J. Chem., 52, 1424-1435, 1974.

Westenberg, A. A., and N. DeHaas, Absolute measurements of the O + $\mathrm{C}_{2} \mathrm{H}_{4}$ rate coefficient, Symp. Int. Combust. Proc., 12, 289, 1969.

World Meteorological Organization, (WMO), Atmospheric ozone, Global Ozone Res. and Monitor. Proj. Rep. 16, Geneva, 1985.

Yoshimura, M., M. Koshi, H. Matsui, K. Kamiya, and H. Umeyama, Nonarrhenius temperature dependence of the rate constant for the $\mathrm{H}+\mathrm{H}_{2} \mathrm{~S}$ reaction, Chem. Phys. Lett., 189, 199-204, 1992.

Yung, Y. L., and W. B. DeMore, Photochemistry of Planetary Atmospheres, 194 pp., Oxford Univ. Press, New York, 1999.

Zabarnick, S., J. W. Fleming, and M. C. Lin, Temperature dependence of $\mathrm{CH}$ radical reactions with $\mathrm{H}_{2} \mathrm{O}$ and $\mathrm{CH}_{2} \mathrm{O}$, Symp. Int. Combust. Proc., 21, $713,1988$.

Zellner, R., Recent advances in free radical kinetics of oxygenated hydrocarbon radicals, J. Chem. Phys., 84, 403-407, 1987.

S. K. Atreya and A.-S. Wong, Department of Atmospheric, Oceanic, and Space Sciences, University of Michigan, Ann Arbor, MI 48109-2143, USA. (atreya@umich.edu; aswong@umich.edu)

T. Encrenaz, DESPA, Observatoire de Paris, 92195 Meudon, France. (therese.encrenaz@obspm.fr) 